\title{
THE IMPACT OF OUTWARD REMODELING ON VASODILATION IN SKELETAL MUSCLE RESISTANCE ARTERIES
}

\author{
A Thesis \\ presented to \\ the Faculty of California Polytechnic State University \\ San Luis Obispo
}

\author{
In Partial Fulfillment \\ of the Requirements for the Degree \\ Master of Science in Biomedical Engineering \\ by \\ Ryan Gallagher \\ December 2012
}


(C) 2012

Ryan Gallagher

ALL RIGHTS RESERVED 


\section{COMMITTEE MEMBERSHIP}

TITLE:

AUTHOR:

DATE SUBMITTED:

COMMITTEE CHAIR:

COMMITTEE MEMBER:

COMMITTEE MEMBER:
The Impact of Outward Remodeling on Vasodilation in Skeletal Muscle Resistance Arteries

Ryan Gallagher

December 2012
Dr. Trevor Cardinal, Assistant Professor

Dr. Lily Laiho, Associate Professor 


\author{
ABSTRACT \\ The Impact of Outward Remodeling on Vasodilation in \\ Skeletal Muscle Resistance Arteries \\ Ryan Gallagher
}

Peripheral arterial occlusive disease (PAOD) is an ischemic disease characterized by narrowing of the peripheral arteries due to the accumulation of atherosclerotic plaque in the inner lining of the vessels, which disrupts blood flow to downstream tissues. Blood can be redirected into collateral vessels, natural bypasses around arterial occlusions, causing shear-induced outward remodeling of the vessels. The enlarged vessels facilitate transfer of increased blood flow to downstream tissues. The remodeling process, however, may impair vasodilation, which in turn may cause or contribute to intermittent claudication- transient pain brought on by locomotion. To stimulate the growth of collateral arteries, the femoral arteries of young, otherwise healthy mice were ligated distally to the profunda femoris, the stem to the gracilis collateral circuit. The diameter of the profunda femoris artery was measured at rest and following gracilis muscle contraction 7 and 28 days post-surgery using intravital microscopy. Enlarged resting diameter, consistent with collateral enlargement, and impaired vasodilation was observed at day 7 , but not at day 28 . To determine if impaired functional vasodilation is due to impaired endothelial- or smooth muscle-dependent responses during outward remodeling, cell-dependent vasodilators were applied to the hindlimb. Endothelial- and smooth muscle-dependent vasodilation was significantly impaired 7 days post-ligation, but not 28 days after. This data supports the hypothesis that smooth muscle dysfunction causes impaired functional vasodilation in the early stages of collateral enlargement.

Keywords: Outward Remodeling, Vasodilation, Arteriogenesis, Collateral, Peripheral Arterial Occlusive Disease, Ischemia, Gracilis 


\section{ACKOWLEDGMENTS}

I would like to thank Dr. Trevor Cardinal for his continued support and guidance, the other members of the Microcirculation and Tissue Repair Lab, and my parents for their endless love and support.

"The more that I see the less that I know for sure."

John Lennon 


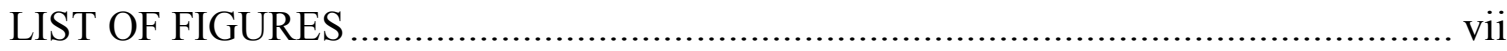

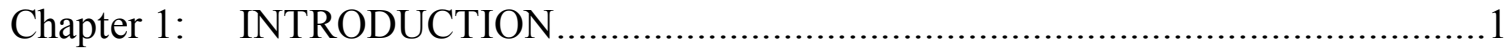

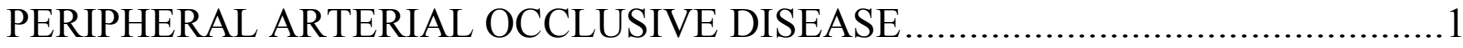

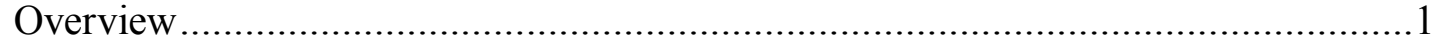

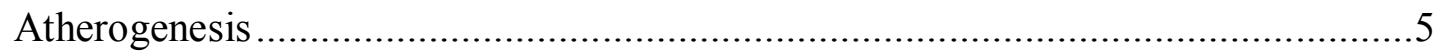

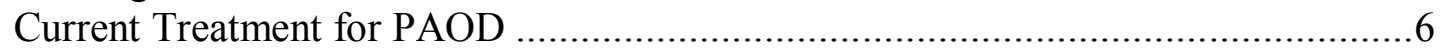

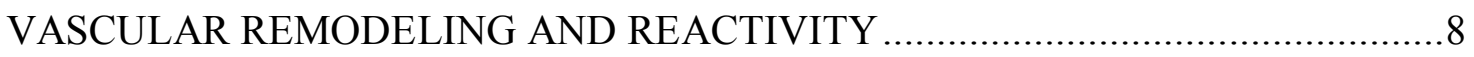

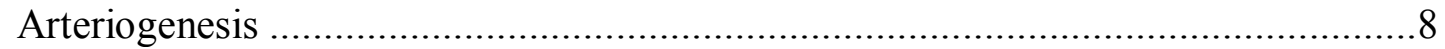

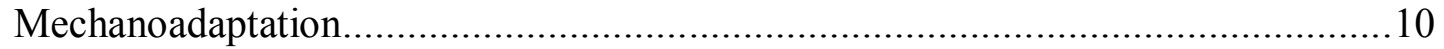

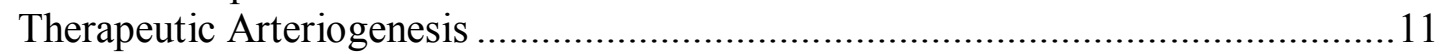

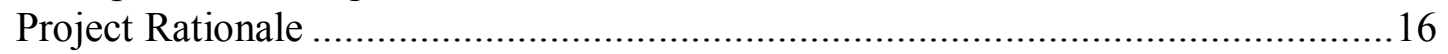

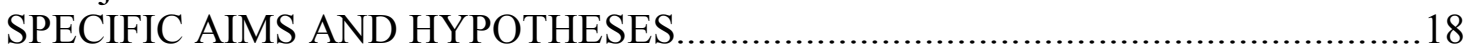

Chapter 2. FUNCTIONAL VASODILATION PROTOCOL VERIFICATION ..........20

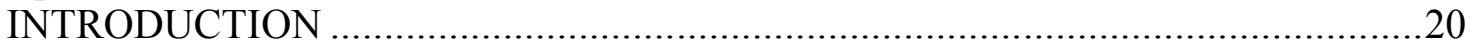

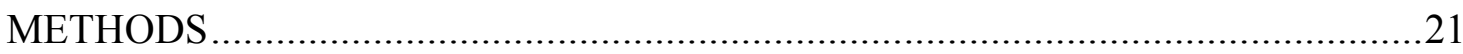

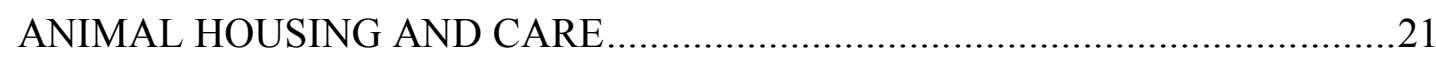

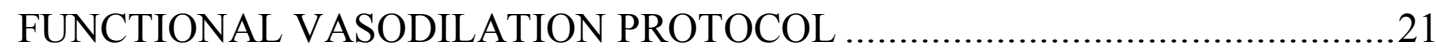

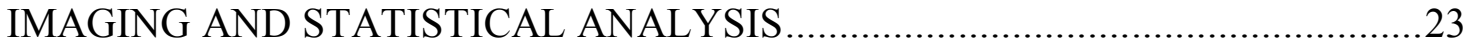

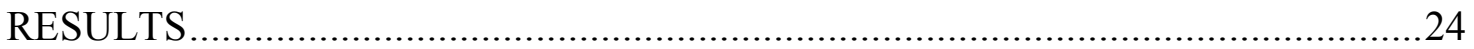

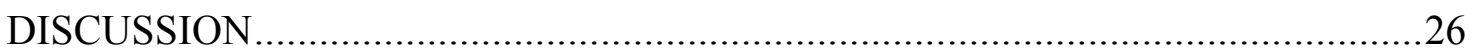

Chapter 3: FUNCTIONAL VASODILATION FOLLOWING FEED ARTERY

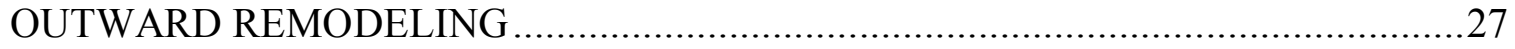

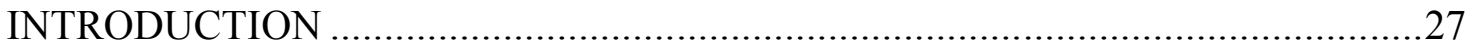

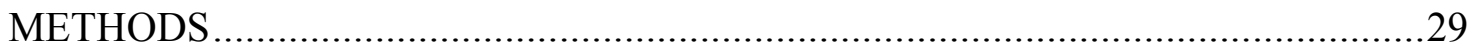

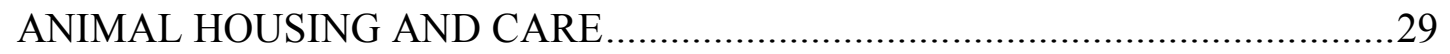

FEMORAL ARTERY LIGATION PROTOCOL.................................................29

FUNCTIONAL VASODILATION WITH INTRAVITAL MICROSCOPY ............32

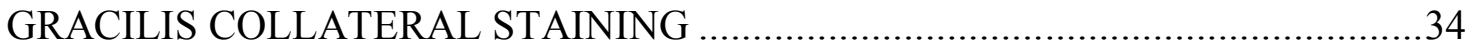

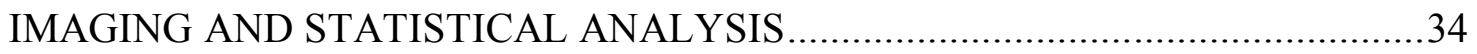

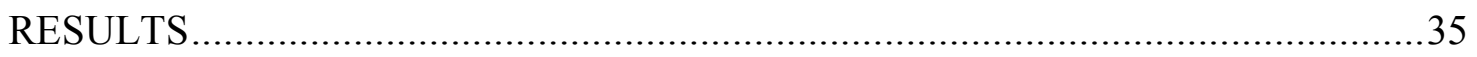

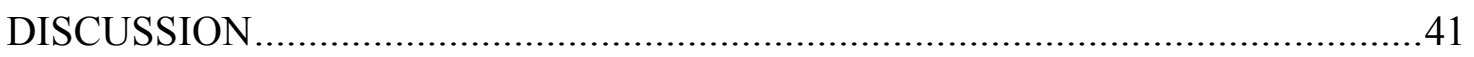

Chapter 4: VASCULAR REACTIVITY WITH INTRAVITAL MICROSCOPY .......46

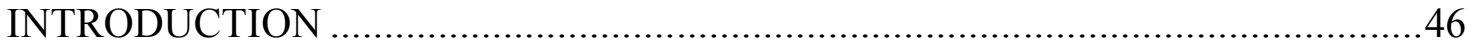

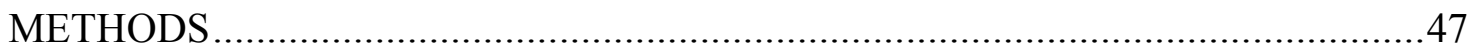

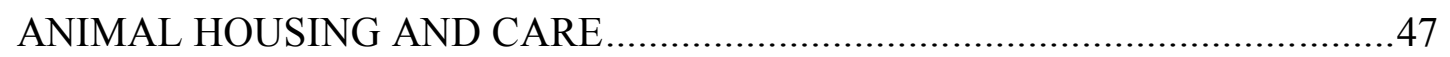

FEMORAL ARTERY LIGATION PROTOCOL .................................................4

VASCULAR REACTIVITY WITH INTRAVITAL MICROSCOPY .....................48

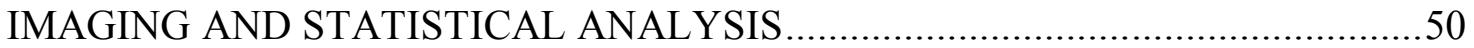

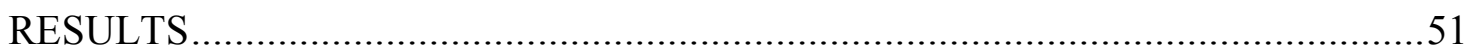

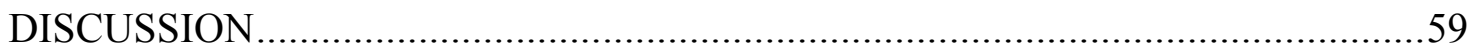

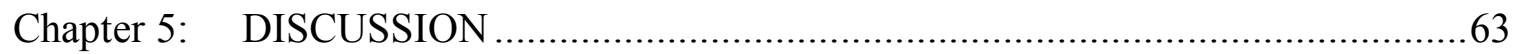

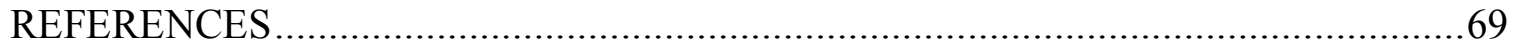

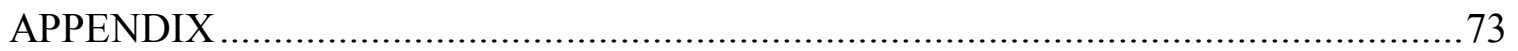




\section{LIST OF FIGURES}

Figure 1: Peripheral Arterial Occlusive Disease...............................................

Figure 2: Hemodynamic Changes Induced by Arterial Occlusion ...............................5

Figure 3: Arteriogenesis in a Collateral Circuit .................................................... 9

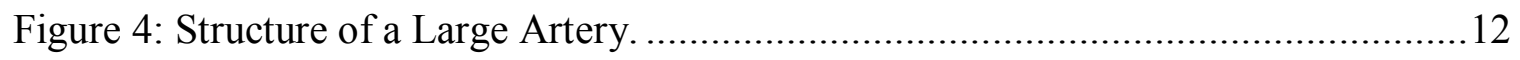

Figure 5: Vasodilatory Mechanisms.......................................................... 14

Figure 6: Vasodilation in Normal and Inflammatory Conditions. ...............................15

Figure 7: The Mouse Hindlimb Collateral Circuit. .................................................17

Figure 8: Stimulating Microelectrode Placement.................................................23

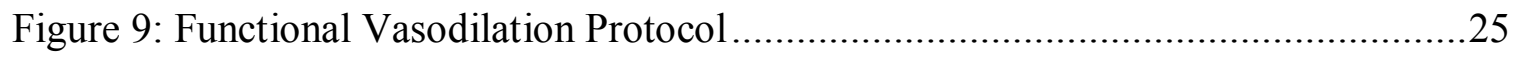

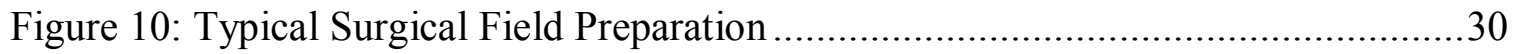

Figure 11: Mouse Hindlimb with Ligation Site ............................................. 31

Figure 12: Progression of the Femoral Ligation Surgery ...................................... 32

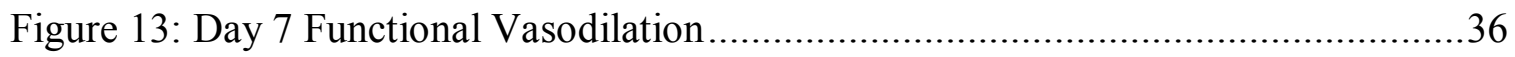

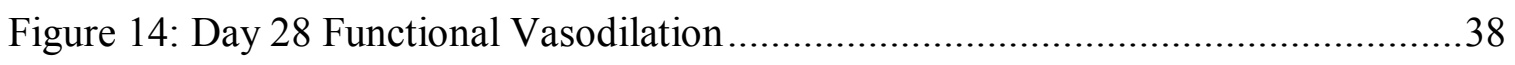

Figure 15: Collateral Arteriole Diameter ..................................................... 40

Figure 16: Superfusion Experimental Setup ................................................ 49

Figure 17: Day 7 Acetylcholine Reactivity. ....................................................52

Figure 18: Day 7 Sodium Nitroprusside Reactivity ..............................................54

Figure 19: Day 28 Acetylcholine Reactivity ..................................................56

Figure 20: Day 28 Sodium Nitroprusside Reactivity ........................................58

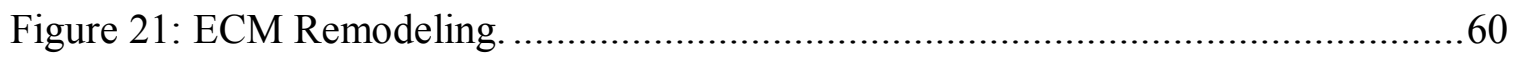




\section{Chapter 1: INTRODUCTION}

\section{PERIPHERAL ARTERIAL OCCLUSIVE DISEASE}

Overview

Peripheral arterial occlusive disease (PAOD) is an ischemic disease characterized by narrowing of the peripheral arteries due to the accumulation of atherosclerotic plaque in the inner lining of the vessels. The accumulated plaque obstructs blood flow to downstream tissues, causing ischemia, an insufficient blood flow to match metabolic demand, which results in tissue hypoxia and potentially necrosis.

There are an estimated 5 to 12 million adults in the United States affected by PAOD and this number will likely increase as the population ages $[1,2]$. Blockages within the peripheral arteries of these individuals lead to diminished blood flow to the extremities (Figure 1). Ischemia-induced claudication (ischemic pain induced by locomotion) can result and if the disease progresses further, blood flow can diminish enough to necessitate amputation. The presence of PAOD in patients suggests a decrease in overall cardiovascular health as they are more likely to develop blockages in blood vessels elsewhere in the body. PAOD is a reliable predictor of coronary heart disease (CHD) and cerebrovascular disease (CBVD), which can lead to myocardial infarction and stroke [3]. The manifestation of atherosclerotic occlusions in other vascular beds is the major determinant of mortality in patients with PAOD; the ten-year mortality rate for cardiovascular events is three- to six-fold greater in patients with PAOD [4]. 


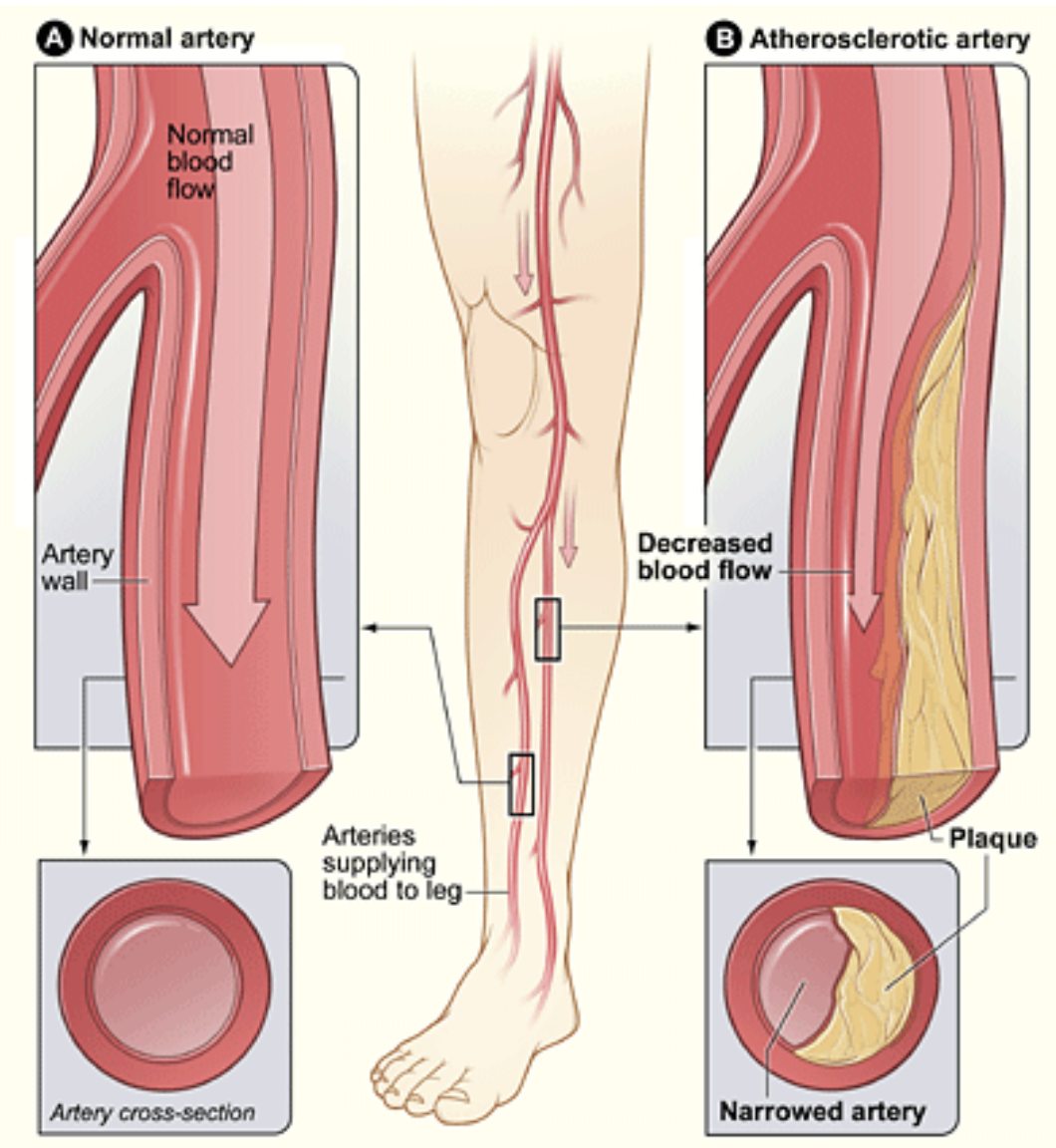

Figure 1: Peripheral Arterial Occlusive Disease. PAOD is characterized by arterial narrowing due to atherosclerotic plaque buildup [5].

The most common symptom of PAOD is intermittent claudication, or transient limb pain associated with exercise that is relieved by rest, which affects $10 \%$ to $35 \%$ of patients [1]. Evaluating the distance a patient can walk until experiencing pain is the gold-standard method of quantifying the severity of intermittent claudication. Intermittent claudication is associated with a 2- to 4-fold increased risk of mortality, mainly from cardiovascular disease $[6,7]$. Forty to fifty percent of patients experience critical limb ischemia, a more severe form of PAOD in which leg pain does not correlate with exertion and is not relieved by rest due to severe blockage of vessels [6]. Gangrene or ulceration 
present in approximately $2 \%$ of patients as a result of critical limb ischemia [1]. In extreme cases, the blockage of blood flow and nutrients to downstream vessels can be severe enough to necessitate amputation. Interestingly, most individuals with PAOD experience no symptoms relating to the disease, which further exacerbates the risk of mortality from PAOD and CHD as individuals are unaware of the need for treatment [1]. Furthermore, diagnosis of PAOD is difficult as unlike CHD, which typically presents as angina, asymptomatic PAOD diagnosis requires uncommon instrumentation.

The Ankle Brachial Index (ABI) is the most commonly used diagnostic tool for evaluating a patient for PAOD as it is simple and noninvasive. Using a continuous wave Doppler device, the ratio of blood pressure in the posterior tibial artery with respect to the brachial artery is determined [1]. Occlusion of the peripheral arteries results in a decrease in blood pressure in the legs. By comparing the ratio of blood pressure in the lower leg to that in the arm, the severity of arterial blockage can be quantified. A normal ABI is 0.9 to 1.3 , suggesting blood pressure in the leg is approximately the same or greater than the pressure in the arm. ABI values of 0.41 to 0.90 indicate mild to moderate PAOD while values under 0.40 indicate severe PAOD [1]. In addition to being used to diagnose PAOD, abnormal ABI values are independent predictors of morbidity and mortality [8]. Treadmill exercise testing is coupled with pre- and post-exercise ABI measurements in patients who are highly suspect to have PAOD, but exhibit normal ABI values [1]. Other methods of diagnosing PAOD include non-invasive techniques such as Doppler ultrasonography, computed tomographic angiography, and magnetic resonance angiography [1]. A more invasive technique, catheter based angiography, involves the 
injection of a contrast agent into the vasculature. Catheter based angiography is helpful in analyzing the impact of PAOD on hemodynamics [1].

The major risk factors for PAOD are similar to the risk factors for coronary heart disease and include advanced age, a family history of the disease, African-American heritage, and male sex [1]. Controllable risk factors include hypertension, hyperlipidemia, diabetes, and smoking [1], of which smoking and diabetes are the strongest and may accelerate PAOD [4].

The etiology of PAOD is similar to other ischemic diseases, such as coronary heart disease, and involves atherogenesis induced by endothelial injury and inflammation [1]. The risk factors for atherogenesis in the peripheral arteries induce endothelial damage, which initiates the buildup of atherosclerotic plaque. This buildup restricts the flow of blood from the heart to the periphery, resulting in ischemic injury in downstream tissues, which become dependent on the collateral circulation (Figure 2). 

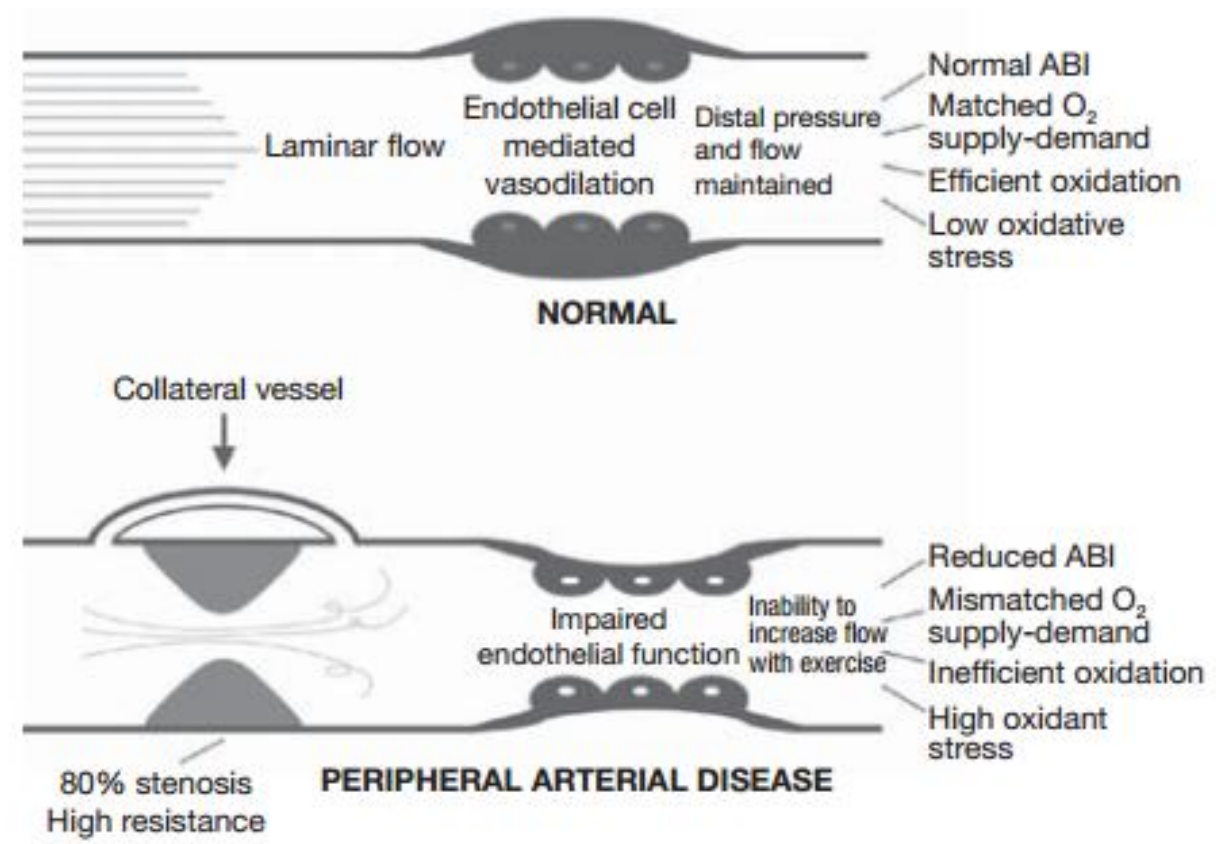

Figure 2: Hemodynamic Changes Induced by Arterial Occlusion. Illustrations of an artery and the properties of the downstream vasculature and tissue in the absence (top) and presence of (bottom) peripheral arterial occlusion. Adapted from [9].

Atherogenesis

Atherosclerotic lesions are comprised of three major components: cellular material (primarily smooth muscle cells and macrophages), connective tissue (primarily extracellular matrix), and an intracellular component comprised of lipids within macrophages and foam cells [10]. The pathogenesis of atherosclerosis begins with turbulent flow within a vessel as illustrated by the observation that atheromas tend to occur at vessel bifurcations, areas of disturbed flow patterns $[10,11]$. In response to this turbulence, endothelial cells become activated, form intercellular gaps, and present adhesion molecules on their surface through degranulation of intracellular vesicles [10]. Molecules such as VCAM-1 are presented on endothelial cell surfaces and bind to monocytes and T-cells [11], which extravasate through the gaps between the activated 
endothelial cells. Macrophage-colony stimulating factor induces monocytes to transform into macrophages and begin to phagocytose oxidized LDL (ox-LDL) [11]. This process is thought to be a protective measure to minimize endothelial exposure to inflammatory oxLDL, but chronically elevated ox-LDL stimulates T-cell/macrophage interactions and a positive feedback cycle of inflammation. T-cells, activated by ox-LDL antigens presented by the macrophages, secrete inflammatory cytokines such as interferon- $\gamma$, which further activates endothelial cells and macrophages [11]. Macrophages secrete factors that initiate the proliferation and migration of smooth muscle cells that eventually form the neointima [11]. Continual phagocytosis of oxidized LDL by macrophages transforms them into foam cells, which also inhabit the neointima [11]. The neointima gradually enlarges to become an atherosclerotic plaque, and occludes the vessel, resulting in ischemia downstream of the occlusion.

\section{Current Treatment for PAOD}

As patients with PAOD are likely to experience atherosclerosis in vascular beds other than those in the extremities, the current treatment approach focuses on both improvement of lower limb ischemia and reduction of overall cardiovascular risk, and includes lifestyle changes, drug therapy, and revascularization procedures [4]. The first course of action in treatment of PAOD is risk factor modification through lifestyle changes such as exercise, diet change, and cessation of smoking. When PAOD is diagnosed early, lifestyle changes can prevent disease progression.

Exercise and smoking cessation cause the greatest improvement in patients with PAOD. Exercise therapy improves risk factors for PAOD, including lipid profile, glucose metabolism, and blood pressure [12]. Walking distance until the presentation of 
claudication is increased with exercise [12] and increased peripheral artery wall thickness (a predictor of atherosclerosis) can be reversed through exercise training [13]. The mechanism for this effect may be due to restoration of eNOS signaling and improved endothelial structural integrity [14]. Some limitations of exercise are that only supervised therapy has been shown to be effective [12]; patients may not strictly adhere to the training regimen, and insurance companies may deny exercise training coverage. The fact that exercise often elicits claudication may explain poor patient adherence to exercise therapy. Smoking cessation is another lifestyle change that can prevent disease progression. Smoking increases an individual's risk of PAOD by sevenfold and is a more reliable predictor for PAOD than for coronary artery disease [15]. Stopping smoking leads to a ten-year reduction in mortality rate from $54 \%$ to $18 \%$ [16], however, cessation of smoking rates following written or oral recommendation by a doctor are as low as $13 \%$ after two years [17].

If lifestyle changes are not enough to improve symptoms in patients, additional treatments are implemented. Antiplatelet therapy to reduce the likelihood of clotting, such as aspirin or Clopidrogel, reduces vascular death in patients with any form of atherosclerosis by approximately $25 \%$ and is equally as effective in treating patients with PAOD as with patients with coronary artery disease [16]. Angiotensin converting enzyme inhibitors and angiotensin receptor blockers, which prevent the production and function of a potent vasoconstrictor, reduce cardiovascular morbidity and mortality in PAOD patients by approximately $25 \%[16]$.

If lifestyle changes or pharmacologic therapies do not sufficiently arrest the progression of $\mathrm{PAOD}$, revascularization procedures are performed. The most commonly 
used endovascular treatment is percutaneous transluminal angioplasty, with or without stent placement [18]. Bypass surgery is performed when long sections of a vessel are atherosclerotic or if a plaque is not suitable for angioplasty. There is no significant difference in outcome of percutaneous angioplasty and bypass surgery after four years [19].

While there are many treatment options available for the management of PAOD, no one course of action is comprehensive to alleviate symptoms. Furthermore, many patients with PAOD are unsuitable candidates for revascularization therapies. Those with heavily calcified lesions, fully occluded arteries, long occlusions $(>5 \mathrm{~cm})$, and lack of suitable vessels for bypass grafting are unable to receive intervention [18]. There is thus significant motivation to develop novel therapeutic strategies to improve blood flow, such as by stimulating the growth or formation of collateral arteries- natural bypass vessels.

\section{VASCULAR REMODELING AND REACTIVITY}

Arteriogenesis

PAOD is characterized by the occlusion of arterial vessels which disrupts blood flow to downstream tissues. When a conduit artery becomes occluded by an atherosclerotic plaque, there is a decrease in arterial pressure distal to the occlusion, Figure 3. The disparity between the high arterial pressure proximal to the occlusion and the low arterial pressure distally causes blood flow to be redirected into collateral arterioles connecting the two regions, providing an alternate blood flow path to the circulation downstream of the occlusion. The redistribution of blood flow into the collateral arterioles causes increased flow velocity and shear stress on the vessel wall, which initiates outward remodeling. Outward remodeling of the vessel restores the shear 
stress on the vessel wall to normal values (between 10 to 70 dyne $\mathrm{cm}^{\wedge} 2$ ) and increases blood flow to ischemic tissues via the natural bypasses [20].
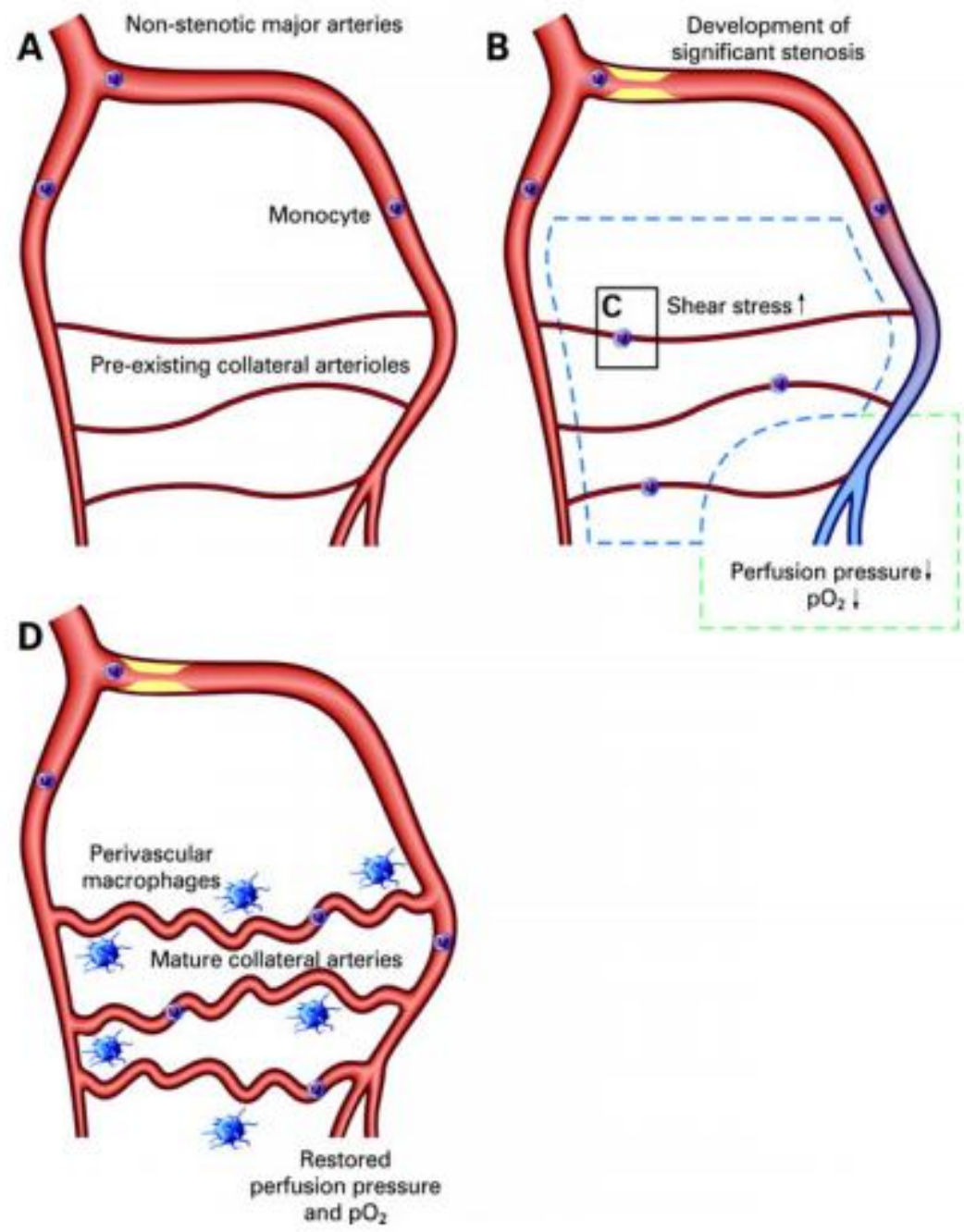

Figure 3: Arteriogenesis in a Collateral Circuit. Conduit artery occlusion leads to a decrease in blood pressure distal to the occlusion. Blood is redirected into collateral vessels, causing them to enlarge [21].

The prognosis of those with cardiovascular disease can be predicted by the presence of collaterals. Coronary collaterals both prevent myocardial ischemia and limit infarct size in patients with coronary occlusions. In patients with PAOD, larger and more 
numerous collateral vessels are associated with better $\mathrm{ABI}$ values and a lower prevalence of asymptomatic PAOD [22], making the stimulation of collateral artery growth a promising and potentially beneficial treatment option for patients with ischemic disease. Outward vessel remodeling can be achieved through two mechanisms, arteriogenesis and mechanoadaptation. Arteriogenesis, discussed above, involves the proliferation of endothelial and smooth muscles cells and degradation of extracellular matrix, while mechanoadaptation involves the non-proliferative reorganization of smooth muscle cells within the vessel wall. It is likely that these processes, as well as vasodilation, are part of remodeling continuum that contributes to blood flow control both acutely and chronically [23].

Mechanoadaptation

The simplest mechanism of outward remodeling involves the reorientation of smooth muscle cells (SMCs) within the vessel wall. SMCs are capable of changing their attachments to other cells and extracellular matrix to move within the media [23]. Cellular repositioning allows wall shear stress to equilibrate without adding additional cells to the vessel wall through proliferation. When continually exposed to a vasoconstrictor, SMCs migrate to increase their overlap with adjacent cells to decrease baseline vessel diameter [24]. While not as well-documented, continual exposure to vasodilatory agents leads to acute outward remodeling, likely due to smooth muscle migration [23]. Due to our poor understanding of the mechanisms of mechanoadaptation, it has not been investigated as a therapeutic strategy. 
Therapeutic Arteriogenesis

While mechanoadaptation is the simplest mechanism of outward remodeling, arteriogenesis, a much more complex process, is better understood and more widely studied. Arteriogenesis is initiated by an increase in shear stress on the vessel wall, which causes activation of the endothelial cells lining the vessel lumen. A signaling cascade involving various cytokines and growth factors is carried out to provide the inflammatory environment needed for outward growth. Endothelial cells and smooth muscle cells divide and matrix metalloproteinases (MMPs) degrade the extracellular matrix. In the later stages of arteriogenesis, remodeling beings [25]. SMCs begin to synthesize a new elastic lamina and the downregulation of MMPs facilitates formation of the intima and remodeling of the media, Figure 4. SMCs begin to migrate into the intima. Some SMCs in the media begin to apoptose and are replaced by new ones. The SMCs that begin to proliferate change their phenotype from a contractile one to a synthetic one. Arteriogenesis results in an enlarged vessel capable of increasing blood flow to downstream tissues. 


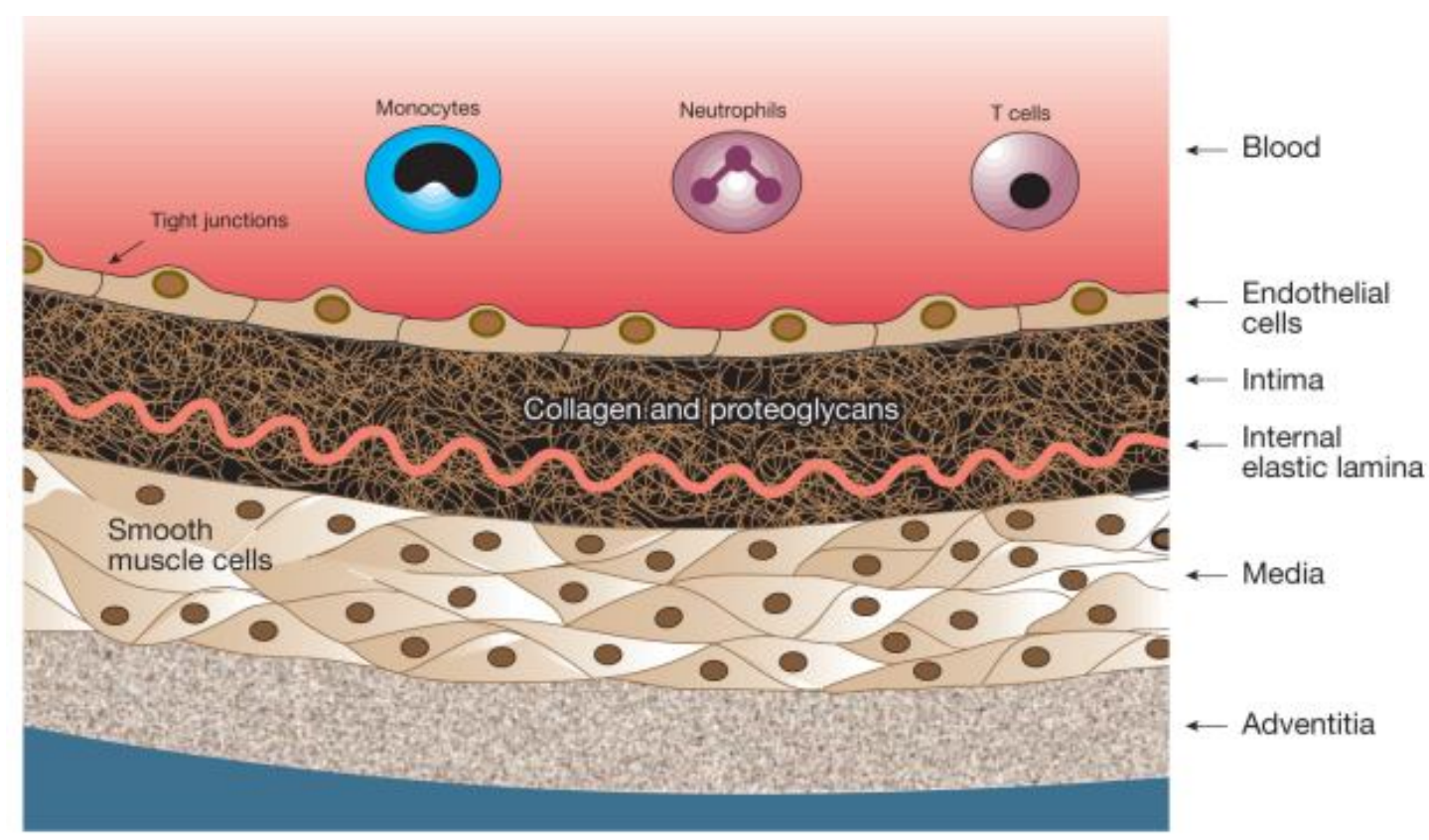

Figure 4: Structure of a large artery. During arteriogenesis the vessel wall is remodeled to normalize the shear stress brought on by increased fluid flow [26].

Therapeutic stimulation of collateral vessel growth is a promising therapy to improve blood flow to ischemic tissue. A variety of factors have been identified as targets to stimulate arteriogenesis, with some reaching clinical testing, and others abandoned in the pre-clinical phase due to adverse side effects. Granulocyte-macrophage colonystimulating factor (GM-CSF), a factor shown to be pro-arteriogenic in the rabbit hindlimb, increased collateral blood flow in clinical trials, but did not improve walking distance to claudication [27]. Fibroblast growth factor, an endothelial and smooth muscle cell mitogen [28], administration increased walking distance to claudication [29] and MCP-1, which stimulates monocyte recruitment, induced collateral growth, but is unsuitable as a therapeutic as it is increases atherosclerotic plaque formation. Transforming growth factor beta-1 (TGF $\beta-1)$, a monocyte chemoattractant, is a promising therapeutic as it is both pro-arteriogenic and atheroprotective. In order for pro- 
arteriogenic therapies to be effective, the enlarged vessels need to maintain vasoreactivity to properly match tissue nutrient demand. During arteriogenesis, however, vascular smooth muscle cells are thought to be poorly reactive due to their shift in phenotype, resulting in impaired vasodilation. In order to develop effective therapies, the mechanisms controlling vasodilation during outward remodeling of vessels need to be better understood.

\section{Remodeling and Vasoreactivity}

Although outward remodeling in collateral arteries could produce a natural bypass around an occluded conduit artery, the remodeling process may impair vascular reactivity- potentially worsening symptoms of intermittent claudication. The ability of a vessel to dilate or constrict is essential to maintain homeostasis by matching the supply of oxygen and nutrients to downstream cellular demand. Without effective blood flow control to tissues, tissue damage can occur due to insufficient nutrient supply and waste product removal. This is especially critical for vessels supplying peripheral skeletal muscle, as increased muscle fiber activity results in increased metabolic demand that is orders of magnitude greater than at rest. In skeletal muscle, hyperemia through vasodilatory mechanisms can manifest from a variety of sources including skeletal muscle fibers, erythrocytes, endothelial cells, and smooth muscle cells, Figure 5. Endothelial cells can also transmit vasodilation signals to adjacent cells via endothelial gap junctions, through a process termed conducted vasodilation [30]. Outward remodeling of the vessels, however, may impair these vasodilatory mechanisms, as previously observed in our lab [31]. 
A

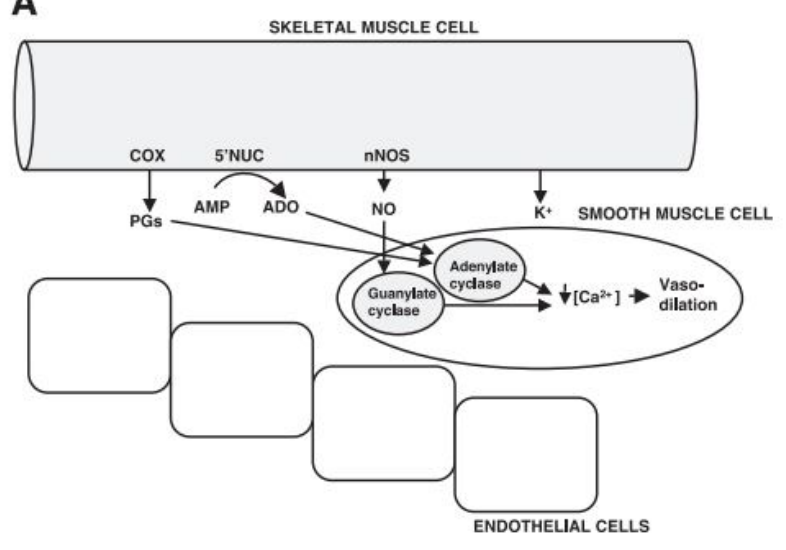

C
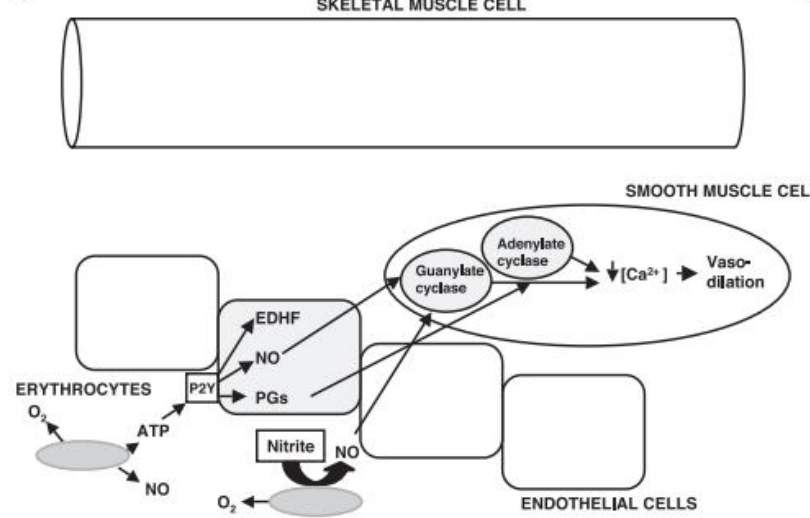

B
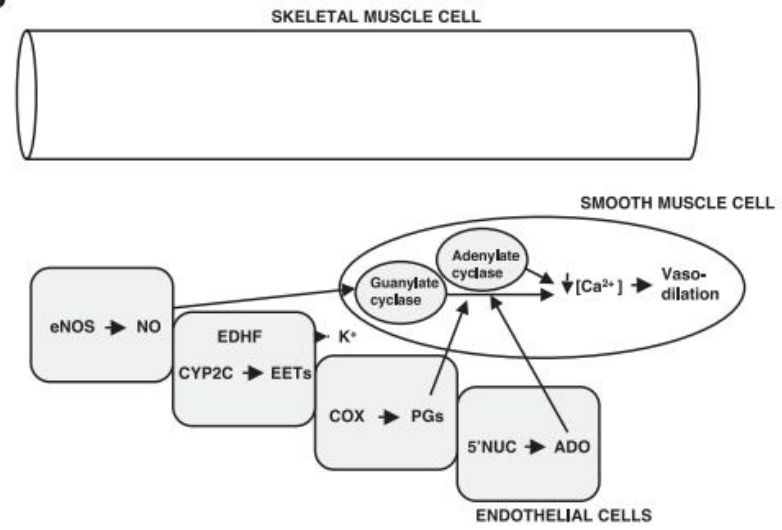

D

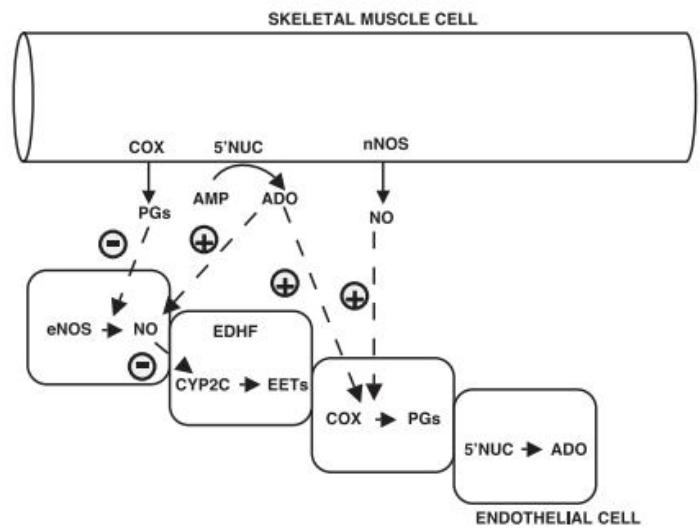

Figure 5: Vasodilatory Mechanisms. Vasodilatory mechanisms originated from (A) skeletal muscle cells, (B) endothelial cells, (C) erythrocytes. Interaction between these systems is shown in (D) [32].

A possible explanation for impaired vasodilation during outward remodeling is the process of cellular repositioning during mechanoadaptation. Mechanoadaptation involves the concerted remodeling of the extracellular matrix-integrin-cytoskeletal axis, which may impair the ability of smooth muscle cells to relax, resulting in smaller dynamic range of the vessel. Another possible explanation for impaired vasodilation during arteriogenesis is the transition of smooth muscle cells from a contractile to a synthetic phenotype, as described above. The loss of contractile proteins and the accumulation of free ribosomes and endoplasmic reticulum associated with this 
phenotype switch may impair the ability of the SMCs to control blood flow via contraction and relaxation. This is likely not the cause of impaired vasodilation in small arteries as no smooth muscle cell proliferation (a trademark of arteriogenesis) is present during outward remodeling of these vessels [33].

Another explanation for impaired vasodilation during outward remodeling is that inflammation, a critical component of both PAOD and arteriogenesis, causes endothelial dysfunction and significantly impacts blood flow. Under normal conditions, endothelial cells induce vasodilation by releasing nitric oxide (NO) in response to receptor-dependent dilators and increased shear stress [34]. During inflammation, however, cytokines activate endothelial NADPH oxidase which produces superoxide [34]. Superoxide inactivates $\mathrm{NO}$ and inhibits the ability of receptor-dependent dilators and shear stress to induce vasodilation, Figure 6.

\section{Control}

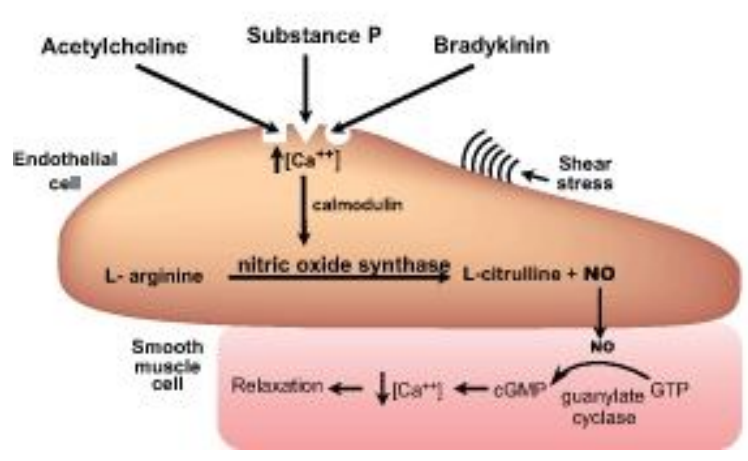

Inflammation

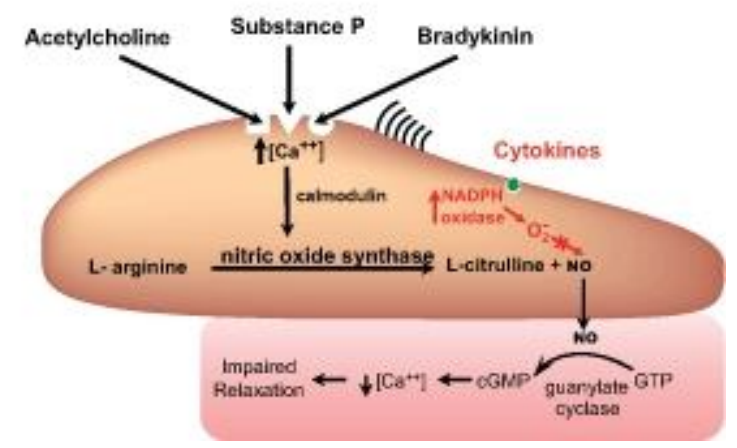

Figure 6: Vasodilation in normal and inflammatory conditions. Vasodilation is impaired during inflammation [34].

It is possible that the mechanisms discussed above are involved in the impaired vasoactivity seen in patients with PAOD, as atherosclerosis and arteriogenesis are 
inflammatory processes. This hypothesis is supported by the observation that nonoccluded vessels in patients with PAOD also exhibit impaired vasoreactivity, suggesting the disease elicits a systemic response. Other factors associated with inflammation such as C-reactive protein, cytokines and oxidized low density lipoprotein (oxLDL) may also be involved in this impairment [35]. Determining which cell types are affected by these factors is critical to developing strategies to improve vasodilation.

Vascular reactivity studies aimed at determining the cell type responsible for the impairment in vasodilation observed in outwardly remodeling vessels are inconclusive, with some studies suggesting impaired endothelial dependent dilation $[36,37]$ and others implicating smooth muscle cells [38].

Project Rationale

Baseline diameter of the profunda femoris feed artery, the stem of the gracilis collateral circuit, increases following femoral artery ligation (Figure 7). Further, functional vasodilation has been observed in our laboratory to be both impaired and normal in the profunda femoris artery following femoral ligation. If functional vasodilation is impaired following femoral artery ligation, it suggests that the outward remodeling process of the profunda impairs its ability to dilate. Previous studies observed no smooth muscle proliferation in the vessel, suggesting that the impairment in vasodilation during outward vascular remodeling (if confirmed to be present) is due to the reorientation of smooth muscle cells (mechanoadaptation) [33]. An analogous impairment in vasoreactivity occurs with inward remodeling following prolonged exposure to a vasoconstrictor [24]. 


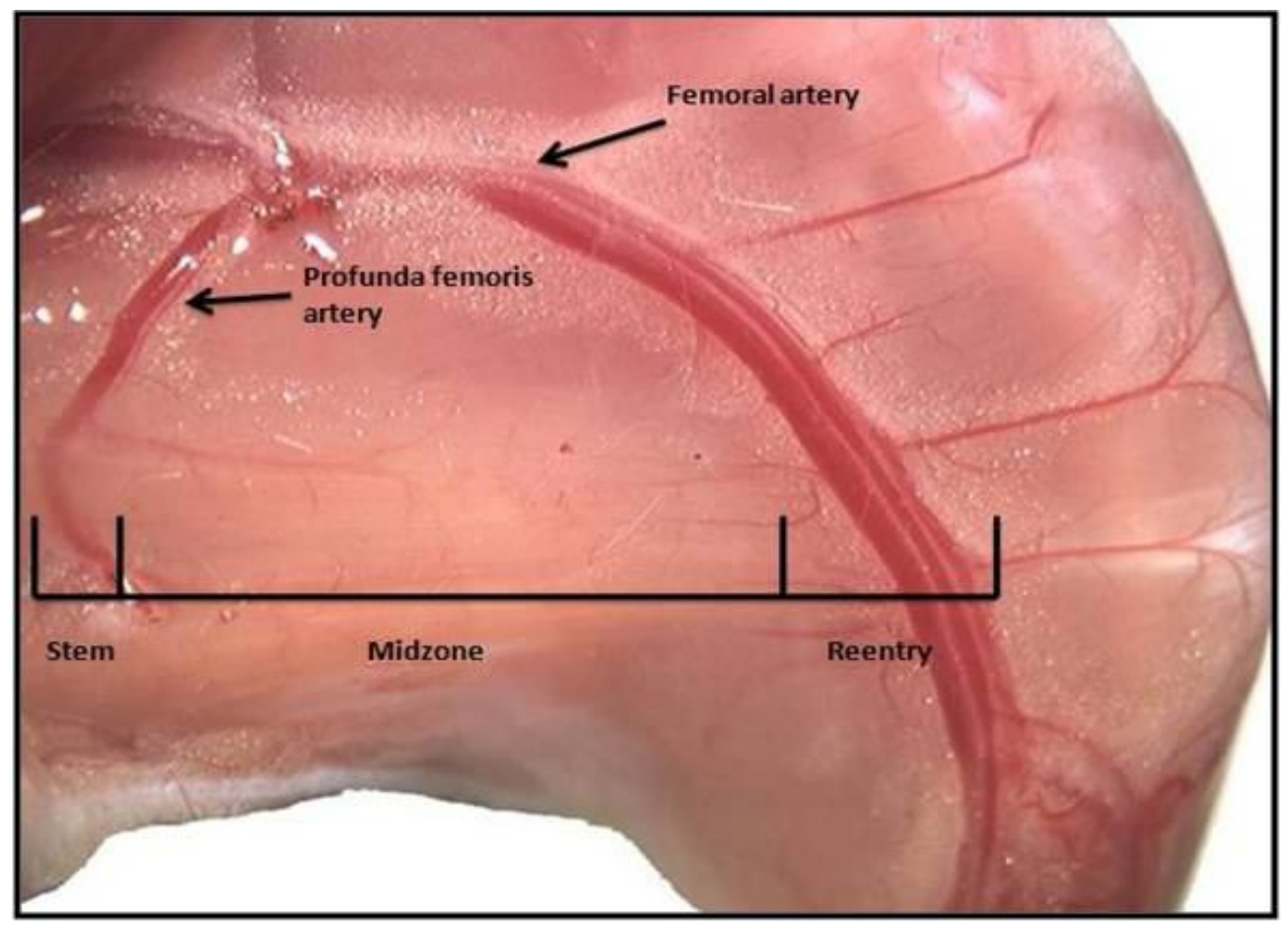

Figure 7: The Mouse Hindlimb Collateral Circuit. Collateral vessels originating from the profunda femoris artery anastomose with the femoral artery. 


\section{SPECIFIC AIMS AND HYPOTHESES}

The overall goal of this research is to determine how outward arterial remodeling impacts vasodilation in the stem region of the collateral circuit. Understanding the effect of outward remodeling on vasodilation could eventually lead to the development of more effective therapeutic agents for patients with peripheral arterial occlusive disease by determining if endogenous collateral enlargement or exogenous stimulation of vessel growth impairs vasodilation.

Specific Aim 1: To test the hypothesis that functional vasodilation of the profunda femoris artery is due to increased metabolism in contracting muscles.

The purpose of this aim is to ensure that any vasodilation observed in the profunda femoris artery is a result of an increase in gracilis metabolism with electrical stimulation of muscle contraction, and not due to direct electrical stimulation of the vessel itself, as was suggested by reviewers of a grant submitted by our lab.

Specific Aim 2: Test the hypothesis that outward vascular remodeling results in a transient impairment in functional vasodilation.

The purpose of this aim is to validate previous research. I expect that vasodilation will be impaired at the early stages of outward remodeling, 7 days post-femoral ligation, but will be restored following the outward remodeling process, 28 days post-ligation. 
Specific Aim 3: Test the hypothesis that outward vascular remodeling results in a transient impairment in smooth muscle-dependent vasodilation.

The purpose of this aim is to determine if impaired functional vasodilation is due to impaired endothelial- or smooth muscle-dependent responses during outward remodeling. 


\section{Chapter 2. FUNCTIONAL VASODILATION PROTOCOL VERIFICATION}

\section{INTRODUCTION}

Vasodilation transiently increases the diameter of a vessel, resulting in increased blood flow to downstream tissues, and is critical for maintaining homeostasis. Without proper vasodilation, metabolically active tissues are unable to receive sufficient oxygen and nutrients to match cellular demand, resulting in tissue damage. The effects of impaired vasodilation manifest as intermittent claudication in patients with PAOD and a better understanding of this impairment is needed to identify therapeutic targets to treat this dysfunction.

In this study, the gracilis muscle is stimulated to induce endogenous vasodilation of the profunda femoris. Stimulation of the motor end plates innervating the gracilis muscle leads to muscle contraction, which causes an increase in metabolic activity and subsequent vasodilation of the muscle feed artery due to conducted vasodilation, the transmission of vasoactive signals to adjacent cells through gap junctions. Vasodilation results from relaxation of the smooth muscle cells within the vascular wall due to decreased intracellular calcium concentration.

To ensure that vasodilation of the profunda femoris, or alterations to that vasodilation response following arterial occlusion, are a result of endogenous vasodilation and not direct electrical stimulation of the vessel, vasoreactivity of the profunda was assessed following quadriceps stimulation, a muscle near, but not fed by the profunda. It is hypothesized that stimulation of a muscle not fed by the profunda femoris will have no effect on the vasoactivity of the vessel due to the highly directional conducted vasodilation response. 


\section{METHODS}

ANIMAL HOUSING AND CARE

Male C57B1/6 mice from Taconic Farms aged 7-9 weeks were used for all experiments according to protocols reviewed and approved by the Cal Poly State University Institutional Animal Care and Use Committee. Mice were housed in a temperature controlled room within the University Vivarium, with four mice per microisolator cage. Nesting material, a "mouse house", and a plastic tube were provided as enrichment. Mice were monitored daily, given water and rodent chow ad libitum, and maintained on a 12 hour light- 12 hour dark cycle.

\section{FUNCTIONAL VASODILATION PROTOCOL}

Mice were anesthetized in an induction chamber with 5\% isoflurane in oxygen flowing at $\sim 31 \cdot \mathrm{min}^{-1}$. The animal was then weighed and transferred to a preparatory bench where anesthesia was reduced to $\sim 1-3 \%$ isoflurane in oxygen flowing at $0.8-1.0$ $1 \cdot \mathrm{min}^{-1}$. Hindlimb hair was removed with clippers and depilatory cream. The mouse was then placed supine on a heated surgical stage. Body temperature was monitored by a rectal thermistor and maintained at $\sim 35^{\circ} \mathrm{C}$ with a heat pad.

A skin incision was made on the middle, medial aspect of the hindlimb and extended proximally to the abdominal wall. Phosphate buffered saline (PBS) was applied to the tissue to prevent desiccation and connective tissue was gently blunt dissected to maximize the tissue exposed. The epigastric fat pad and neurovascular bundle were resected using a heat cautery. Two tungsten microelectrodes (one stimulating and one ground) were rested on either the gracilis muscle (functional vasodilation) or the 
quadriceps muscle (sham) (Figure 8). To ensure correct placement of the microelectrodes to induce strong muscle contraction, a brief set of muscle contractions was performed with $1 \mathrm{~mA}$ square waves $200 \mu \mathrm{s}$ in duration at $1 \mathrm{~Hz}$ using a PowerLab acquisition system. Excess PBS was removed with a KimWipe and mineral oil was placed over the tissue with a pipette to prevent tissue desiccation and oxygen diffusion into the muscle. Plastic wrap was placed over the mineral oil to prevent runoff. An intravital microscope (Olympus BXFM) was positioned over the distal profunda femoris, just before the arteryvein pair passes under the gracilis anterior. The preparation was given 30 minutes to equilibrate. After equilibration, the muscle was stimulated for 90 seconds with $1 \mathrm{~mA}$ square wave $200 \mu$ s in duration at $8 \mathrm{~Hz}$. Images of the profunda femoris were captured before stimulation, immediately following stimulation, and every subsequent minute until the vessel returned to baseline diameter. The gracilis and quadriceps muscles in both hindlimbs were stimulated in random order. Following the experiment, mice were euthanized by cervical dislocation. 


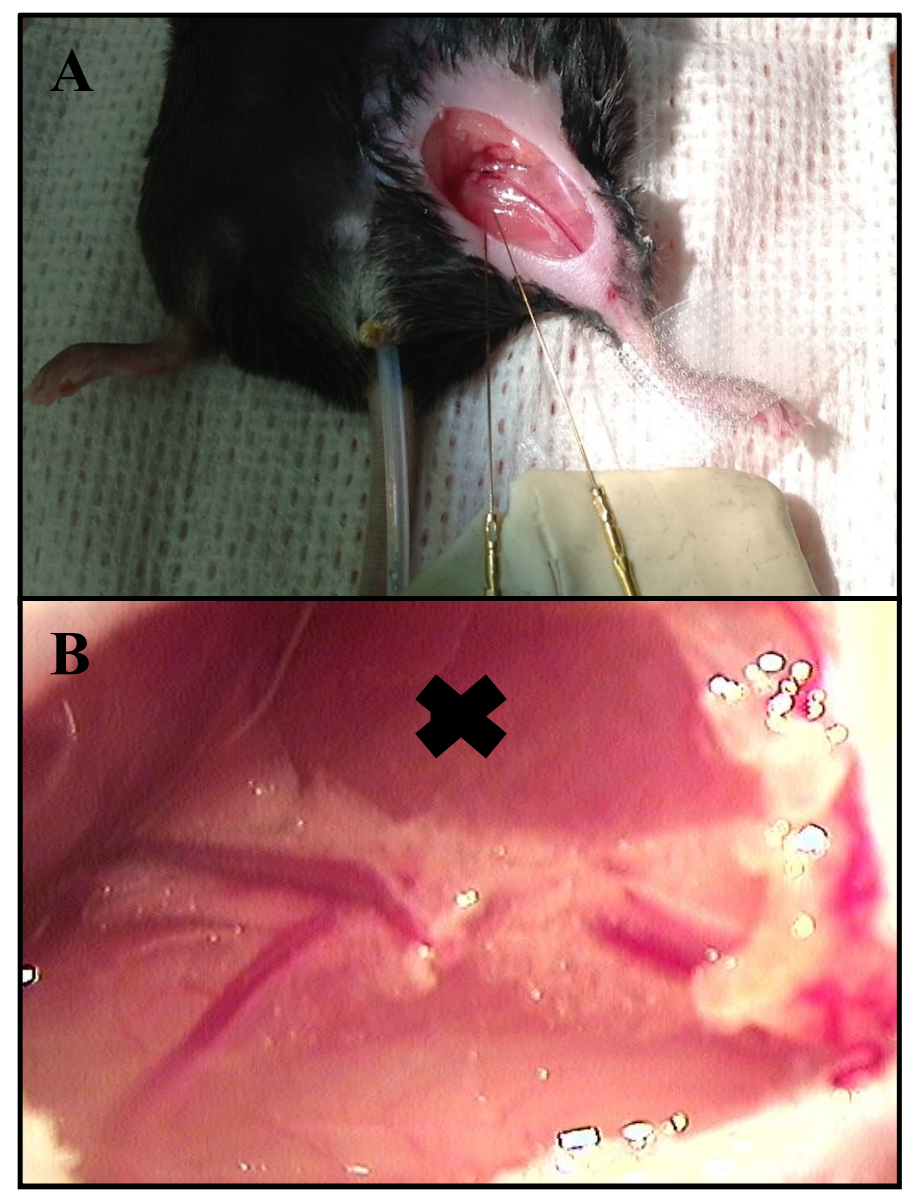

Figure 8: Stimulating Microelectrode Placement. (A) Placement of tungsten microelectrodes on the gracilis muscle and (B) location of electrode placement (X) on the quadriceps during electrical stimulation.

\section{IMAGING AND STATISTICAL ANALYSIS}

Infinity imaging software was used to capture images, ImageJ was used to measure vessel diameters, and Microsoft Excel was used to perform statistical analysis. Data are presented as mean \pm SEM with $\mathrm{p} \leq 0.05$ representing statistical significance. Differences in profunda diameter between muscle groups and pre-/post-contraction were assessed by independent t-test and paired t-test, respectively. 


\section{RESULTS}

Data obtained from the right and left hindlimbs was combined into a single data set after confirming they were not statistically different $(\mathrm{p}>0.40)$. The absolute diameter of the profunda femoris artery after gracilis stimulation was significantly higher than at rest, while the quadriceps diameter remained unchanged (Figure 9C). The percent change of the vessel following gracilis stimulation was significantly higher than after quadriceps stimulation (Figure 9D). 


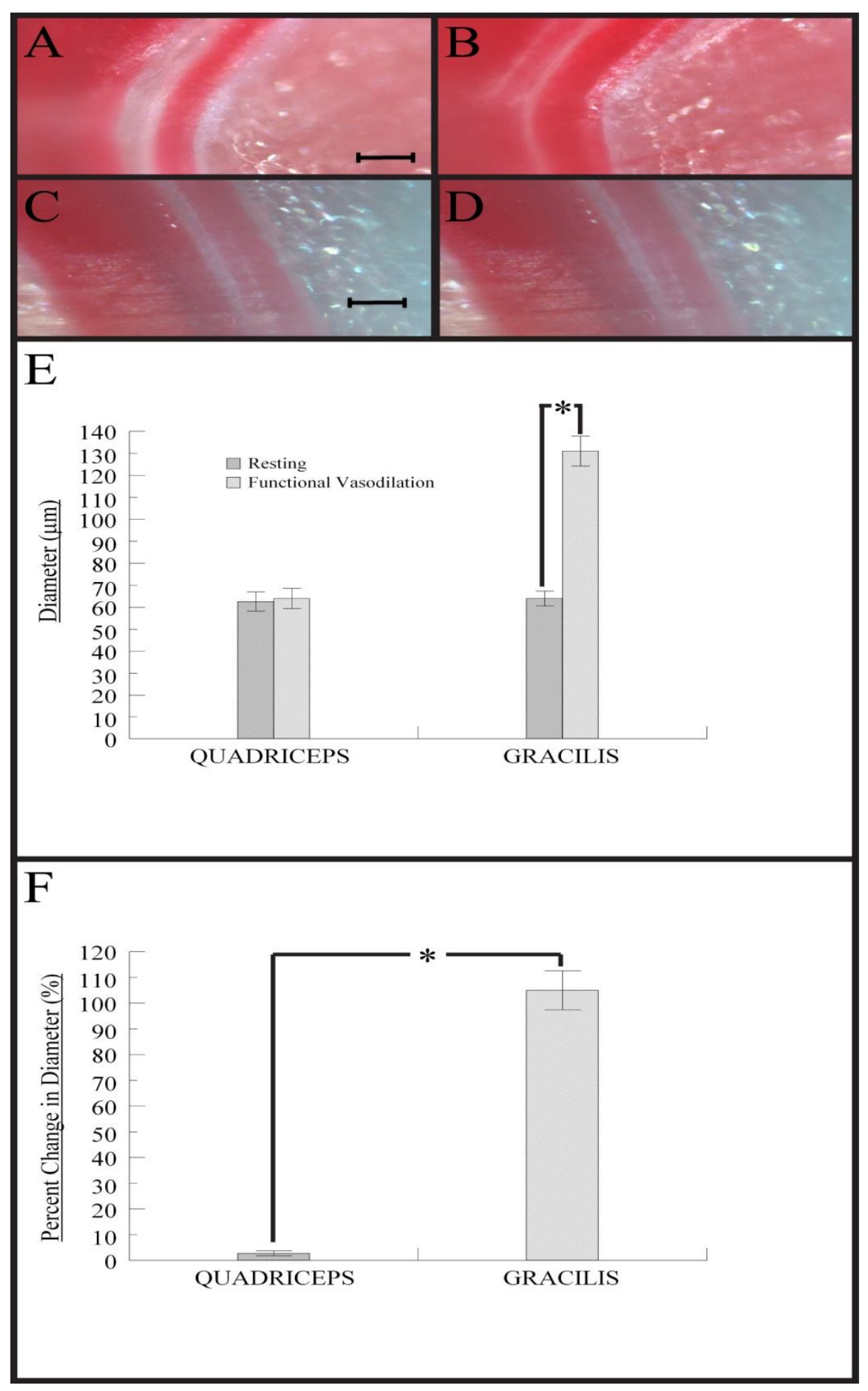

Figure 9: Functional Vasodilation Protocol. (A) Representative images of the profunda femoris artery before and (B) after gracilis stimulation and (C) before and (D) after quadriceps stimulation. (E) The absolute diameter of the vessel following gracilis stimulation was significantly larger than at rest $(\mathrm{p}<0.001)$. (F) Gracilis stimulation elicited a significantly larger percent change in vessel diameter $(p<0.001)$ when compared to quadriceps stimulation $\left(\mathrm{n}_{\text {gracilis }}=8, \mathrm{n}_{\text {quadriceps }}=14\right)$. Scale bar is $100 \mu \mathrm{m}$. 


\section{DISCUSSION}

To verify that vasodilation of the profunda femoris following gracilis stimulation is due to increased metabolism associated with muscle contraction and not direct stimulation of the vessel wall, the quadriceps muscle, a muscle near, but not fed by the profunda was stimulated. The diameter of the profunda femoris was unaffected by quadriceps stimulation, indicating that any impairment in vasodilation is not due to direct electrical stimulation of vasoconstriction by the electrical field (Figure 9). The diameter of the vessel following gracilis stimulation, however, increased dramatically (Figure 9). This indicates that vasodilation of the profunda femoris is due to an increase in muscle metabolism. If stimulation of the muscles did directly affect the cells within the vascular wall, the profunda femoris would have changed diameter in response to quadriceps stimulation. These results suggest that the functional vasodilation protocol employed in the lab is a valid, physiologically relevant method of assessing vasoreactivity of the profunda femoris. This protocol was used for all subsequent functional vasodilation studies (Chapter 3). 


\section{Chapter 3: FUNCTIONAL VASODILATION FOLLOWING FEED ARTERY OUTWARD REMODELING}

\section{INTRODUCTION}

Peripheral arterial occlusive disease is associated with the buildup of plaque within the vascular wall, which not only structurally impedes blood flow, but also impairs the downstream vasculature's ability to dilate, resulting in ischemic injury in downstream tissues. Collateral vessels can act as natural bypasses around the occluded vessel, and the redistribution of blood flow into these vessels causes an increase in blood velocity and wall shear stress, which initiates outward remodeling of the vessel. The enlarged vessel facilitates transport of an additional amount of blood to the ischemic tissues.

The ability of collateral vessels to outward remodel, however, is often impaired in patients with diabetes, hypercholesterolemia, and old age (co-morbidities of PAOD) [3941]. Furthermore, vasodilation may be impaired during outward remodeling as a result of the transition of smooth muscle cell phenotype from contractile to synthetic during arteriogenesis, the repositioning of the cells during mechanoadaptation, or shear-induced inflammation. Impaired vasodilation could then manifest as intermittent claudication, transient pain during exercise, in patients with PAOD. Stimulating outward vascular remodeling via biologic or pharmacologic agents is a promising therapy for patients with PAOD. However, for these therapies to be effective, their effects on vasoreactivity of the vessels must be understood.

This study focuses on the impact of arterial occlusion and subsequent outward collateral remodeling on vasodilation in skeletal muscle resistance arteries. Using the functional vasodilation protocol developed in Chapter 2, the reactivity of the profunda 
femoris was assessed. It was hypothesized that vasodilation would be impaired 7 days post-femoral ligation (early in the remodeling process), but not 28 days after (once remodeling is expected to be roughly complete). 


\section{METHODS}

ANIMAL HOUSING AND CARE

Male C57B1/6 mice from Taconic Farms aged 7-9 weeks were used for all experiments according to protocols reviewed and approved by the Cal Poly State University Institutional Animal Care and Use Committee. Mice were housed in a temperature controlled room within the University Vivarium, with four mice per microisolator cage. Nesting material, a "mouse house", and a plastic tube were provided as enrichment. Mice were monitored daily, given water and rodent chow ad libitum, and maintained on a 12 hour light- 12 hour dark cycle.

\section{FEMORAL ARTERY LIGATION PROTOCOL}

To stimulate the outward remodeling of collateral arteries in the mouse hindlimb, the femoral artery was ligated. Mice were anesthetized and prepared as previously described. Prior to surgery, the mouse was given buprenorphrine analgesic $(0.075$ $\mathrm{mg} \cdot \mathrm{kg}^{-1}$, subQ) and veterinary ophthalmic ointment was placed over the eyes to prevent corneal desiccation. Surgical field preparation is shown in Figure 10. 


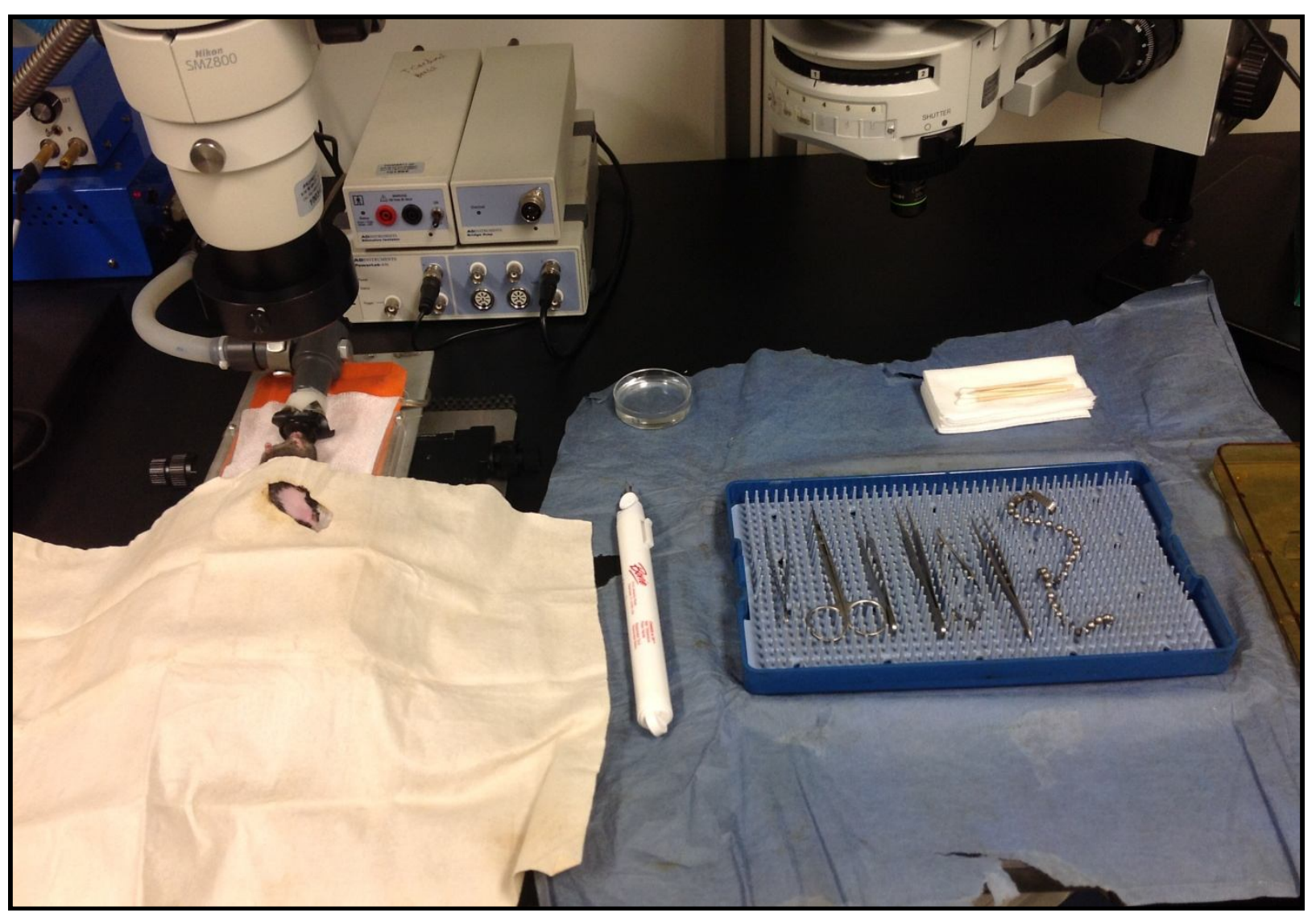

Figure 10: Typical Surgical Field Preparation. The drape, cotton swabs, suture, ligature, and surgical instruments are sterilized prior to the procedure.

Using aseptic technique, a small incision was made on the middle, medial aspect of the left hindlimb, directly over the femoral neurovascular bundle. The incision was extended proximally to the abdominal wall. The area was continually irrigated with sterile saline throughout the procedure to prevent desiccation. Connective tissue was gently blunt dissected and a heat cautery was used to remove the epigastric fat pad and the majority of the epigastric neurovascular bundle. The femoral artery was then dissected from its paired vein and nerve and ligated just distal to the profunda femoris 
branch using 6.0 silk suture (Figure 11). The skin incision was closed using 7.0 polypropylene suture. A diagram of the surgery is shown in Figure 12.

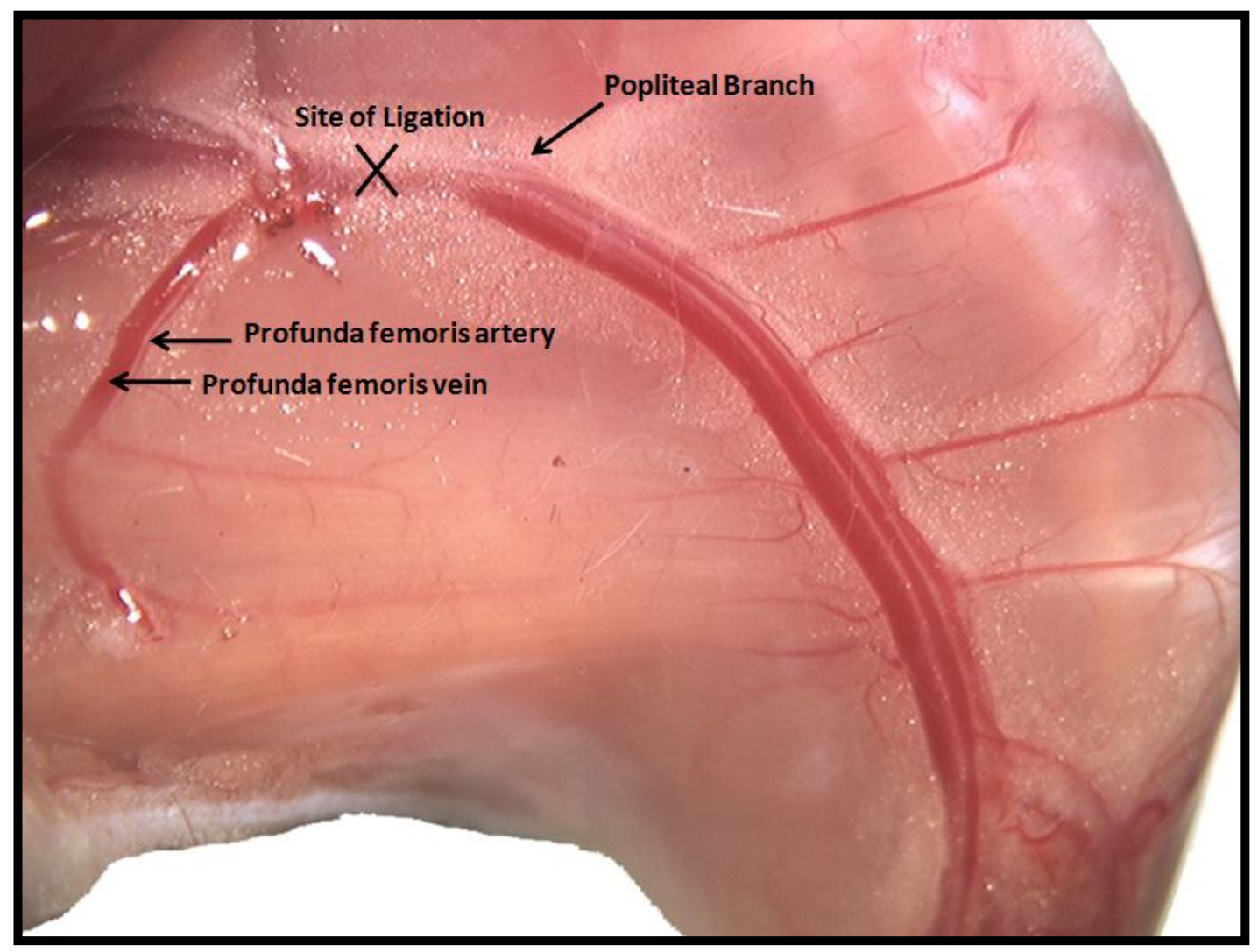

Figure 11: Mouse Hindlimb with Ligation Site. Structure of typical hindlimb vasculature with femoral artery ligation site shown.

A skin incision of similar length and placement was made on the contralateral limb. Sterile saline was applied to the tissue, connective tissue was gently blunt dissected, and the incision was closed with 7.0 polypropylene suture. The contralateral limb was treated as a sham in all experiments to control for the impact of surgical trauma on vasodilation. 
A second dose of buprenorphrine analgesic was given immediately following surgery, as described above, and the animal was allowed to recover with warming until ambulatory.

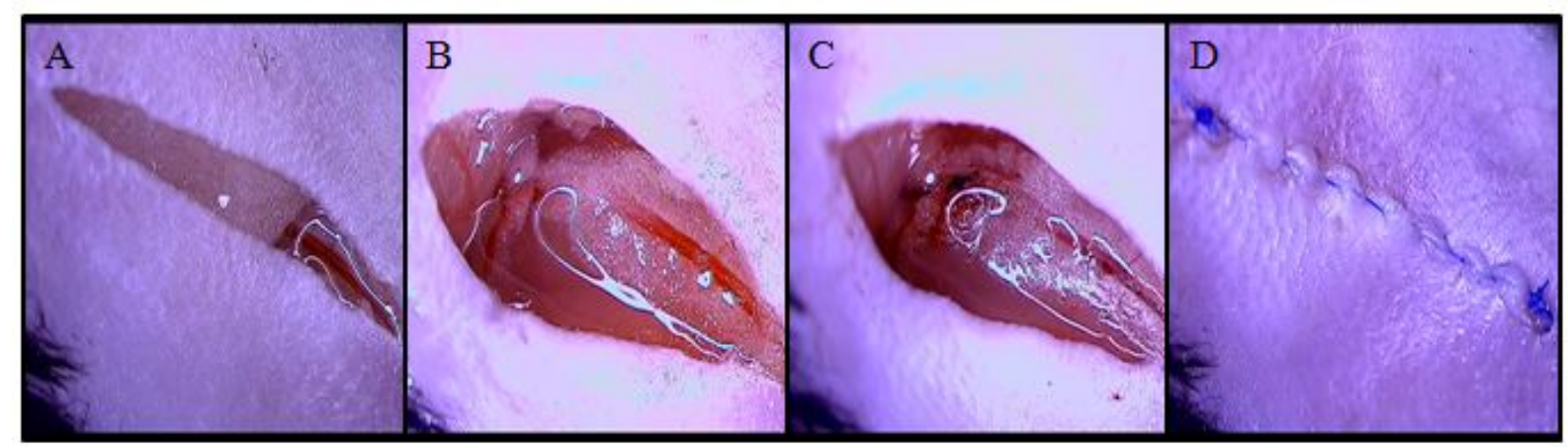

Figure 12: Progression of the Femoral Ligation Surgery. (A) The initial skin incision is made, (B) connective tissue is blunt dissected, and the epigastric fat pad and neurovascular bundle removed. (C) The femoral artery is then ligated distal to the profunda femoris and (D) the incision is closed with 7.0 suture.

\section{FUNCTIONAL VASODILATION WITH INTRAVITAL MICROSCOPY}

At day-7 or 28 after femoral artery ligation animals were anesthetized and the hindlimbs were prepared as described above. A skin incision was made on the medial hindlimb and was extended proximally to the abdominal wall. Phosphate buffered saline (PBS) was applied to the tissue to prevent tissue desiccation and connective tissue was gently blunt dissected. Any newly formed adipose was resected using a heat cautery. Two tungsten microelectrodes (one stimulating and one ground) were rested on the gracilis muscle. To ensure correct placement of the microelectrodes to induce strong muscle contractions, a brief set of muscle contractions was performed with $1 \mathrm{~mA}$ square waves $200 \mu$ s in duration at $1 \mathrm{~Hz}$ using a PowerLab acquisition system. Excess PBS was removed with a KimWipe and mineral oil was pipetted onto the tissue to prevent tissue desiccation and oxygen diffusion into the muscle. Plastic wrap was placed over the mineral oil to 
prevent runoff. The same procedure was followed on the contralateral limb, with the exception that the epigastric fat pad and neurovascular bundle were resected using a heat cautery.

An intravital microscope (Olympus BXFM) was positioned above the distal profunda femoris, just before the artery-vein pair passes under the gracilis anterior, and the preparation was given 30 minutes to equilibrate. After equilibration, the gracilis muscle was stimulated for 90 seconds with $1 \mathrm{~mA}$ square wave $200 \mu \mathrm{s}$ in duration at $8 \mathrm{~Hz}$. Images of the profunda femoris were captured before stimulation, immediately following stimulation, and each subsequent minute until the vessel returned to baseline diameter. Measurement order between the hindlimbs was randomized. Following the experiment, mice were euthanized by cervical dislocation.

\section{GRACILIS ANTERIOR DISSECTION}

Gracilis adductor muscles were dissected from both hindlimbs at day 7 and 28 . The anterior and posterior gracilis muscles were first separated using blunt dissection. The anterior gracilis muscle was then blunt dissected away from the underlying tissue. Paraformaldehyde (4\%) was pipetted onto the tissue and a twenty minute waiting period was observed to allow for tissue fixation. The femoral artery and profunda femoris artery were then cauterized distally and proximally to the gracilis anterior to prevent blood loss. The anterior gracilis was excised by cutting the muscle laterally to the femoral artery and medially to the profunda femoris. The muscle was then placed in PBS and stored at $4^{\circ} \mathrm{C}$. 


\section{GRACILIS COLLATERAL STAINING}

Sylgard-coated plates were prepared at least three days prior to staining. A 10:1 sylgard elastomer: curing agent solution was mixed in a $50 \mathrm{~mL}$ conical tube. From this solution, $500-750 \mu \mathrm{L}$ was added to each well of a 24 well plate. The mixture was allowed to harden at room temperature for 48 hours.

Each excised gracilis anterior muscle was removed from PBS and placed in one of the wells. An antibody solution containing 1:200 1A4 clone (alpha-smooth muscle actin, $\mathrm{Cy} 3$ conjugate) in $0.1 \%$ saponin, $2 \%$ BSA in PBS was prepared. The muscles were incubated in the antibody solution for 72 hours at $4^{\circ} \mathrm{C}$. Muscles were washed in $0.1 \%$ saponin in PBS three times for 20 minutes then with plain PBS for 30 minutes. The plate was wrapped in Parafilm and covered with foil during each wash to prevent evaporation and exposure to light. The muscles were then placed on a slide and cover slipped with mounting media. The slides were stored at $4{ }^{\circ} \mathrm{C}$ in foil prior to imaging with a fluorescent widefield microscope (Olympus BX41).

\section{IMAGING AND STATISTICAL ANALYSIS}

Infinity imaging software was used to capture images from functional vasodilation experiments, QCapture software was used to image dissected and stained muscles, ImageJ was used to measure vessel diameters, and Microsoft Excel was used to perform statistical analysis. Data are presented as mean \pm SEM with $\mathrm{p} \leq 0.05$ representing statistical significance. Differences in sham and experimental vessel diameter were assessed by an independent t-test. 


\section{RESULTS}

Day 7

Baseline diameter of the profunda femoris artery was significantly larger 7 days post-ligation when compared to control limbs, indicating outward remodeling of the vessel (Figure 13C). The absolute diameter of the vessel was significantly larger, while the percent change in diameter was significantly smaller in the operated limb after gracilis stimulation (Figure 13C), indicating that the dynamic range of the vessel is reduced following arterial occlusion. 


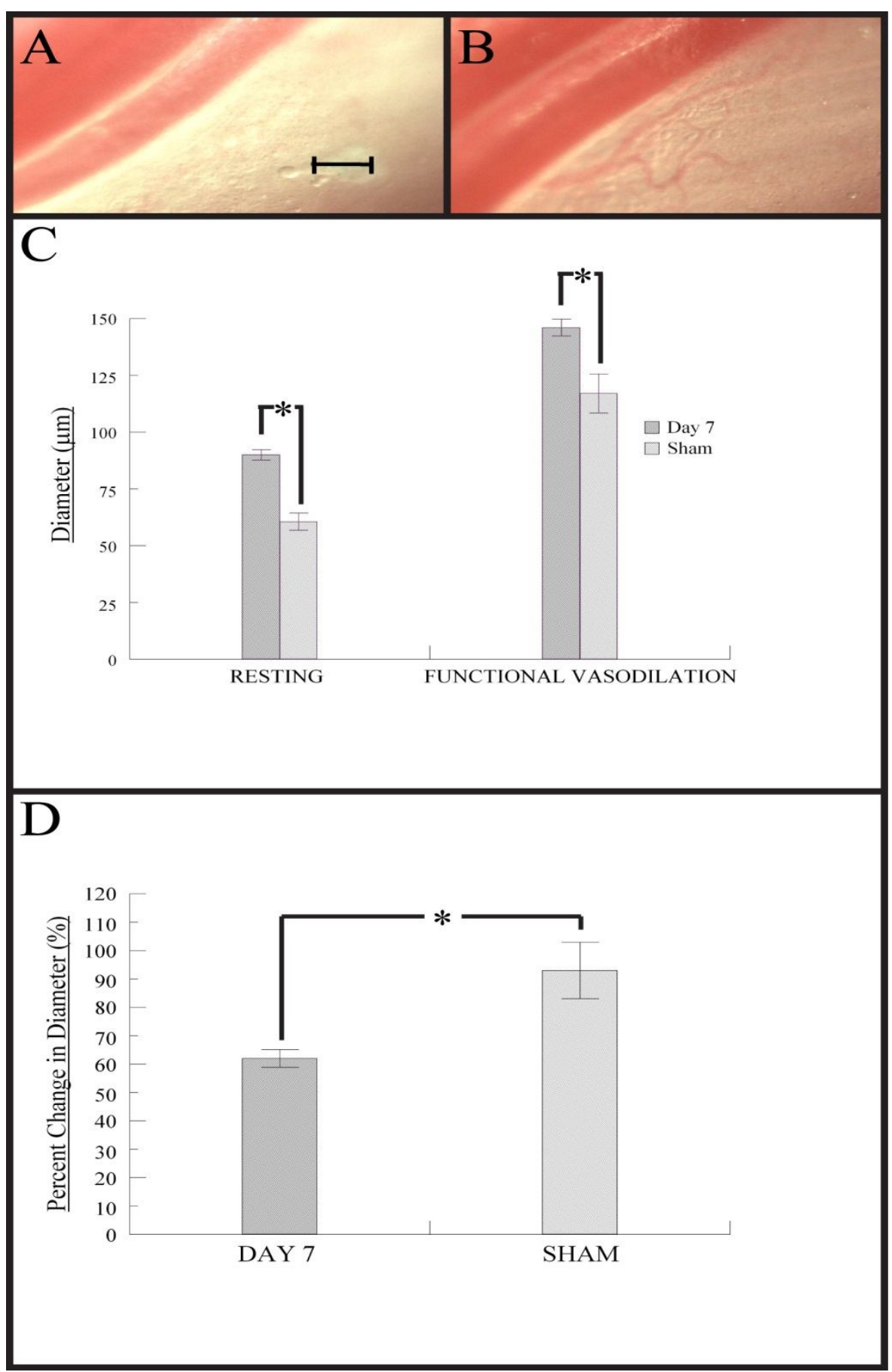

Figure 13: Day 7 Functional Vasodilation. (A) Representative images of the profunda femoris artery in the operated limb before and (B) after gracilis stimulation. (C) The diameter of the profunda femoris in the operated limb was larger at rest and after stimulation ( $p<0.001$ and $p=0.008$, respectively). (D) Percent change in vessel diameter was significantly reduced after femoral ligation $(p=0.02)(n=6)$. Scale bar is $100 \mu \mathrm{m}$. 
Day 28

Since functional vasodilation was reduced 7 days post-ligation, profunda femoris vasoreactivity was assessed after 28 days (a time when vascular remodeling is expected to be complete). Vessel diameter of the profunda femoris at rest and after gracilis stimulation was not significantly different 28 days post-ligation when compared to control limbs (Figure 14C). Percent change in vessel diameter was also not significantly different following femoral ligation (Figure 14D). It was surprising that vessel diameter returned to control values as we expected the diameter to remain larger in order to supply sufficient blood flow to downstream tissue. To assess if the ligation protocol used in these experiments indeed caused long-term outward remodeling of the collateral circuit, the diameters of the collaterals within the midzone of the gracilis circuit were measured. Since profunda diameter returned to normal values, we expected the diameter of the midzone collaterals to increase following ligation. 


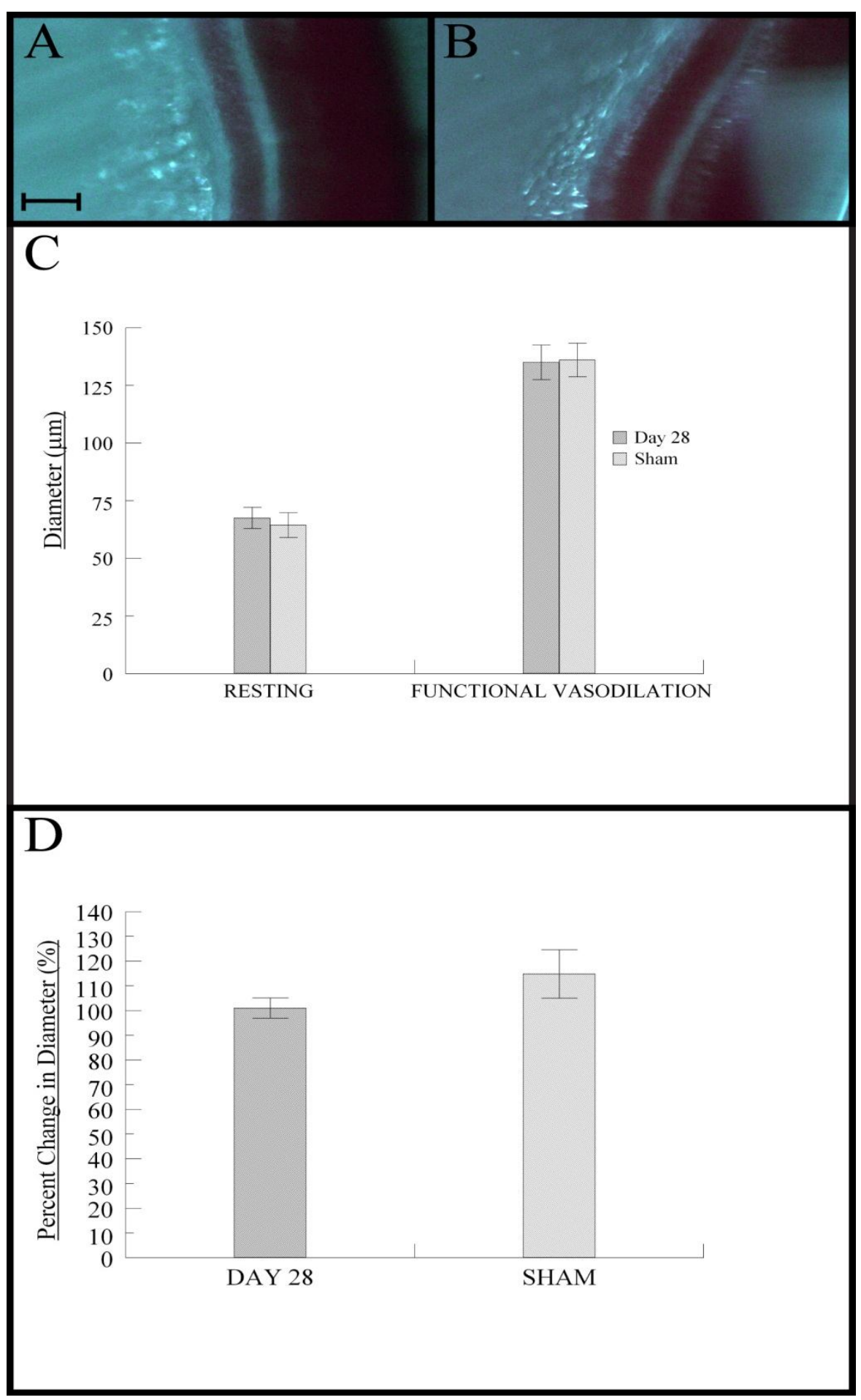

Figure 14: Day 28 Functional Vasodilation. (A) Representative images of the profunda femoris artery in the operated limb before and (B) after gracilis stimulation. (C) The diameter of the profunda femoris in the operated limb was not significantly different at rest or after stimulation ( $p>0.05)$. (D) Percent change in vessel diameter was not significantly different $(\mathrm{p}>0.05)(\mathrm{n}=7)$. Scale bar is $100 \mu \mathrm{m}$. 


\section{Midzone Collaterals}

At both day 7 (Figure 15C) and 28 (Figure 15D), the diameter of the collateral arteriole in the midzone of the gracilis collateral circuit was significantly larger in the ligated limb. This was expected after observing profunda femoris diameter return to control values at day 28 . The increased shear stress within these vessels caused outward remodeling likely via arteriogenesis, allowing blood flow to tissue distal to the ligation site. 


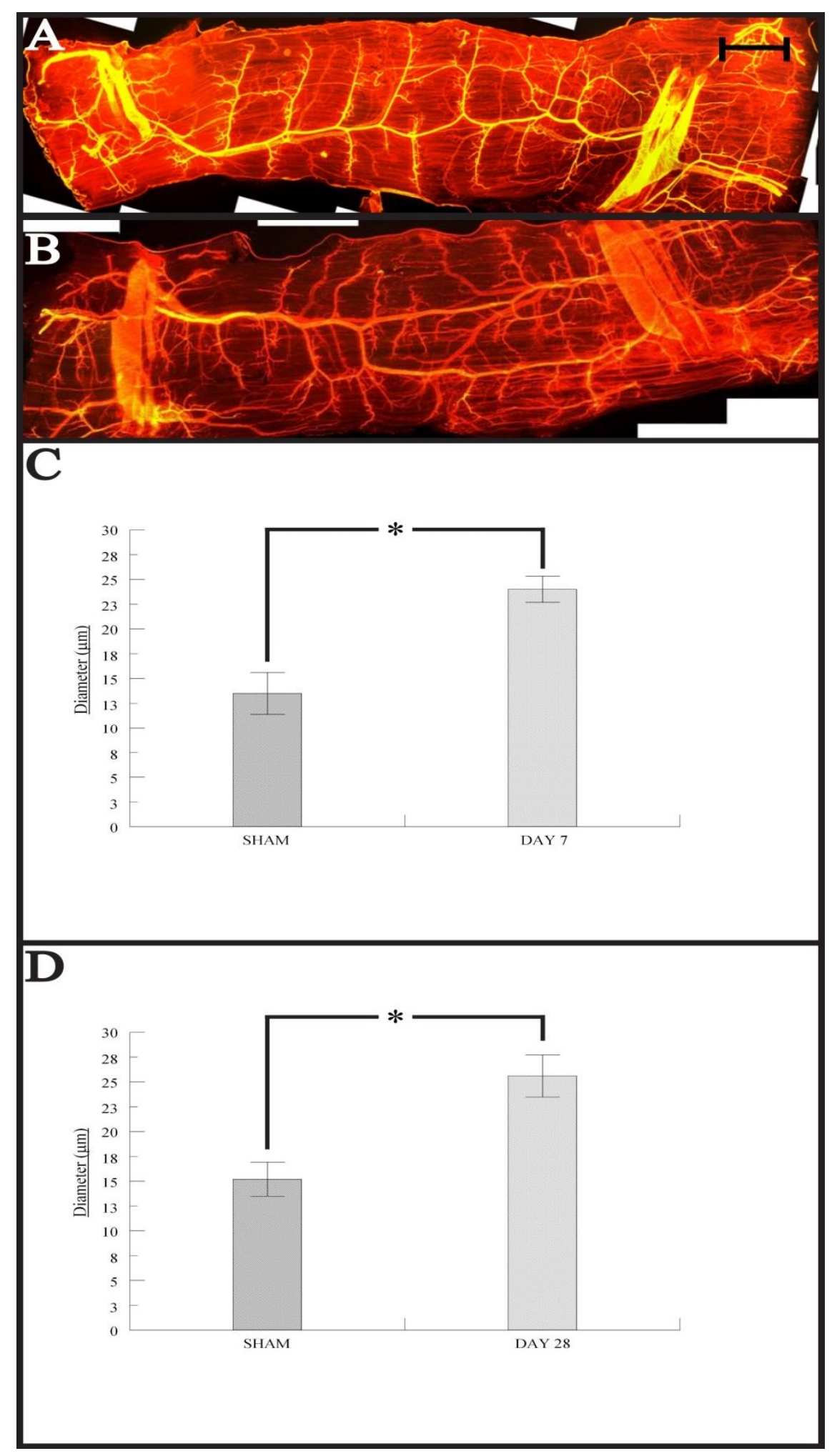

Figure 15: Collateral Arteriole Diameter. Images of alpha smooth muscle actin stained gracilis muscles from the (A) sham and (B) ligated limbs. (C) Arteriole diameter was significantly larger in the ligated limb at $(\mathrm{C})$ day $7(\mathrm{p}<0.05, \mathrm{n}=6)$ and $(\mathrm{D})$ day $28(\mathrm{p}<$ $0.05, \mathrm{n}=5$ ). Scale bar is $2 \mathrm{~mm}$. 


\section{DISCUSSION}

Baseline diameter of the profunda in the ligated limb was significantly larger than in the sham, indicating that outward remodeling occurred (Figure 13C). The impairment in functional vasodilation 7 days after femoral ligation indicates that the outward remodeling process impairs the vessel's ability to dilate (Figure 13D). Vessels can enlarge via proliferation or reorientation of the cells within the vessel wall. Arteriogenesis, the best-documented process of vessel enlargement, is associated with increased wall shear stress and cellular proliferation. No proliferation, however, is present in the profunda at this time point, indicating that a different process of enlargement, such as mechanoadaptation, may be involved [33]. Mechanoadaptation involves the nonproliferative reorientation of smooth muscle cells within the vessel wall. Smooth muscle cells may reorient themselves to decrease their overlap with adjacent cells in response to increased blood flow and shear stress, resulting in enlarged vessel diameter. The reorientation process itself may impair vasodilation as smooth muscle cells migrate throughout the vessel wall. Mechanoadaptation likely involves remodeling of the extracellular matrix-integrin-cytoskeletal axis, which may disrupt cellular communication and relaxation, resulting in impaired dilation.

Interestingly, the diameter of the profunda femoris returned to control values by day 28 , indicating that outward remodeling in the stem region of the collateral circuit is a transient process following artery occlusion (Figure 14C). The midzone collaterals, which are known to undergo arteriogenesis (as evidenced by increased diameter and cellular proliferation 7 days after ligation [33]), maintained their larger diameter at this time point (Figure 15D). Furthermore, functional vasodilation was not impaired at day 
28 , indicating that even though baseline diameter of the profunda femoris was smaller than at day 7, the dynamic range of the vessel, and its ability to respond to increased metabolic demand via vasodilation, was restored (Figure 14D).

The outward remodeling process a vessel undergoes is determined by shear stress and vessel size. The profunda femoris likely remodels by mechanoadaptation, indicating that small arteries are able to normalize increased wall shear stress via reorientation of smooth muscle cells within the vessel wall. The smaller midzone collaterals, however, undergo arteriogenesis to normalize increased shear stress via cellular proliferation. Wall shear stress is inversely proportional to the third power of vessel radius. Hence, normalization of increased shear stress in a small artery would require a smaller degree of remodeling when compared to smaller collateral arterioles. By day 28 , however, the profunda femoris returns to control diameter while the collateral arterioles remain enlarged, indicating that shear stress is only transiently elevated in the profunda, but remains elevated in the arterioles. Following femoral occlusion, the pressure gradient across the collateral circuit is increased, resulting in higher blood velocity and shear stress within the vessels. In response to the increased wall shear stress, both the profunda femoris and the collateral arterioles outwardly remodel. As the arterioles undergo arteriogenesis to stabilize wall shear stress, blood pressure is partially restored distal to the occlusion, resulting in a lower pressure gradient across (and lower shear stress within) the arterioles. A "threshold" of shear stress may be needed to cause outward remodeling [42]. The shear stress in the midzone arterioles is likely still above threshold at day 28 . The pressure gradient across the profunda femoris also decreases following arteriole enlargement, likely reducing shear stress levels enough to induce inward remodeling. 
In order to better characterize the factors that determine whether a vessel undergoes arteriogenesis or mechanoadaptation, experiments should be performed to assess blood velocity and shear stress in the stem and midzone regions of the collateral circuit at day 7 and 28 . In order to determine velocity and shear stress profiles, the streak length of jugular-injected microspheres within the collateral circuit would be measured using fluorescent microscopy.

Once the shear stress profiles within the stem region of the collateral circuit are quantified, the next logical experiment to perform would be to study mechanoadaptation in the profunda femoris with confocal microscopy 7 and 28 days after femoral ligation to assess smooth muscle cell orientation. If mechanoadaptation is occurring, orientation of the cells in the ligated limb would differ from those in the sham. A decrease in overlap between smooth muscle cells would likely be observed at day 7 in response to increased shear stress brought on by the redistribution of blood flow through the vessel. By day 28 , the orientation of the smooth muscle cells in the ligated limb would likely be similar to those in the sham.

Once the remodeling mechanism in the stem region of the collateral circuit is understood, the functionality of the midzone and reentry regions of the circuit should be assessed 7 and 28 days after arterial ligation. Multiphoton microscopy in conjunction with a jugular injection of fluorescent compounds would be used to image the vessels deep within the gracilis. The vasodilatory function of collateral arterioles in response to electrical stimulation of the gracilis would likely be hindered at day 7 as the vessels undergo arteriogenesis. If impairment is observed, it is likely attributed to the change in phenotype of the smooth muscle cells from contractile to synthetic. By day 28 , 
arteriogenesis would be complete [33] and there would likely be no impairment in functional vasodilation.

The results of these studies agree with those obtained by previous members of our lab. Significant profunda femoris enlargement was previously observed 14 days after femoral ligation [43], and a trend toward enlargement was observed at day 7 [33]. Studies assessing vasodilation of the profunda femoris are inconsistent, with some indicating significant impairment [44], and others observing a trend toward impairment [43]. Other research groups observed impaired functional vasodilation in chronically ischemic rat hindlimbs $[45,46]$.

The experiments performed do have some limitations. Firstly, the mouse model employed in these experiments does not accurately mimic the progression of atherosclerotic plaque in humans. Arterial occlusion in patients with PAOD occurs chronically, with the exception of thrombosis, as plaque gradually builds up to occlude the vessel, while the femoral artery of mice used in these studies was fully occluded almost instantly. This difference likely causes different flow patterns within the collateral circuit. Secondly, while care was taken when placing the stimulating microelectrodes to ensure strong muscle contraction, variation in contraction strength was observed. Weak muscle contraction could make it appear as though functional vasodilation is impaired due to reduced vasodilatory stimulus. Thirdly, in some cases, adipose tissue obstructed measurement of the profunda femoris diameter, requiring diameter measurements to be taken from small visible sections. The amount of adipose tissue present decreased after mice were switched to a lower protein diet. 
The impairment in functional vasodilation is likely due to dysfunction of endothelial or smooth muscle cells. The next step in determining if these cell types played a role in the observed impairment in vasodilation was to apply cell-dependent dilators to the hindlimb and measure diameter change of the profunda femoris. 


\section{Chapter 4: VASCULAR REACTIVITY WITH INTRAVITAL MICROSCOPY}

\section{INTRODUCTION}

Following arterial occlusion, blood can be redirected into collateral vessels, resulting in increased vessel wall shear stress and outward remodeling. During outward remodeling of the collateral circuit, vasodilation is impaired (Chapter 3), likely due to endothelial or smooth muscle dysfunction. Patients with PAOD often exhibit impaired vasodilation, which may result in intermittent claudication, ischemic-induced pain during locomotion. Some hypotheses to explain impaired vasodilation include the repositioning of smooth muscle cells during mechanoadaptation or the decrease in endothelial-derived nitric oxide due to shear-induced inflammation. Dysfunction of these cell types would impair the ability of a vessel to dilate and constrict normally to supply adequate blood flow to downstream tissues.

To determine which cell-type is responsible for the impaired functional vasodilation in the profunda femoris (Chapter 3), cell specific vasodilators were superfused over the tissue. Both acetylcholine, an endothelial-dependent dilator, and sodium nitroprusside, a smooth muscle-dependent dilator, were used. Acetylcholine is a neurotransmitter released by nerve endings that binds to receptors on endothelial cells, causing the release of vasodilators (such as nitric oxide, endothelial derived hyperpolarizing factor, and prostacyclin [47]), which bind to adjacent smooth muscle cells, resulting in vasodilation. Sodium nitroprusside acts independently of the endothelium by directly donating nitric oxide to smooth muscle cells to cause vasodilation. Knowing which cell-type is dysfunctional is critical to eventually identifying therapeutic targets to improve vasodilation in patients with PAOD. 


\section{METHODS}

ANIMAL HOUSING AND CARE

Male C57Bl/6 mice from Taconic Farms aged 7-9 weeks were used for all experiments according to protocols reviewed and approved by the Cal Poly State University Institutional Animal Care and Use Committee. Mice were housed in a temperature controlled room within the University Vivarium, with four mice per microisolator cage. Nesting material, a "mouse house", and a plastic tube were provided as enrichment. Mice were monitored daily, given water and rodent chow ad libitum, and maintained on a 12 hour light- 12 hour dark cycle.

\section{FEMORAL ARTERY LIGATION PROTOCOL}

Mice were anesthetized and prepared as previously described. Prior to surgery, the mouse was given buprenorphrine analgesic $\left(0.075 \mathrm{mg} \cdot \mathrm{kg}^{-1}\right.$, subQ) and veterinary ophthalmic ointment was placed over the eyes to prevent desiccation.

Using aseptic technique, a small incision was made on the middle, medial aspect of the left hindlimb, directly over the femoral neurovascular bundle. The incision was extended proximally to the abdominal wall. The area was continually irrigated with sterile saline throughout the procedure to prevent desiccation. Connective tissue was gently blunt dissected and a heat cautery was then used to remove the epigastric fat pad and the majority of the epigastric neurovascular bundle. The femoral artery was then dissected from its paired vein and nerve and ligated just distal to the profunda femoris branch using 6.0 silk suture. The skin incision was closed using 7.0 polypropylene suture. 
A skin incision of similar length and placement was made on the contralateral limb. Sterile saline was applied to the tissue, connective tissue was gently blunt dissected, and the incision was closed with 7.0 polypropylene suture. The contralateral limb was treated as a sham in all experiments.

A second dose of buprenorphrine analgesic was given immediately following surgery, as described above, and the animal was allowed to recover with warming until ambulatory.

\section{VASCULAR REACTIVITY WITH INTRAVITAL MICROSCOPY}

On day-7 or 28 post-surgery, mice were anesthetized and hair was removed as described above. A physiological salt solution (PSS) comprised of (in mM) $\mathrm{NaCl}$ 131.9, $\mathrm{KCl} 4.7, \mathrm{MgSO}_{4} 1.17, \mathrm{CaCl}_{2} 2$ and $\mathrm{NaHCO}_{3} 18$ was prepared in a $1 \mathrm{~L}$ volumetric flask. The flask was placed into a $50^{\circ} \mathrm{C}$ water bath and deoxygenated by bubbling with $5 \%$ $\mathrm{CO}_{2}-95 \% \mathrm{~N}_{2}$; a $\mathrm{pH}$ meter was used to confirm a $\mathrm{pH}$ of $\sim 7.4$. A portion of the PSS was transferred to a heated $60 \mathrm{~mL}$ syringe. The solution exited the syringe through approximately one foot of insulated tubing at a flow rate of $\sim 2 \mathrm{~mL} \cdot \mathrm{min}^{-1}$ and temperature of $\sim 35^{\circ} \mathrm{C}$. A skin incision was made on the medial hindlimb and was extended proximally to the abdominal wall. Phosphate buffered saline (PBS) was applied to the tissue to prevent tissue desiccation and connective tissue was gently blunt dissected and any newly formed adipose was resected using a heat cautery. The tubing was positioned so that the PSS flowed over the mouse hindlimb (Figure 16) and a KimWipe was placed on the distal end of the hindlimb to wick excess solution to a reservoir. An intravital microscope (Olympus BXFM) was positioned above the distal profunda femoris, just before the 
artery-vein pair passes under the gracilis anterior, and the preparation was given 30 minutes to equilibrate.

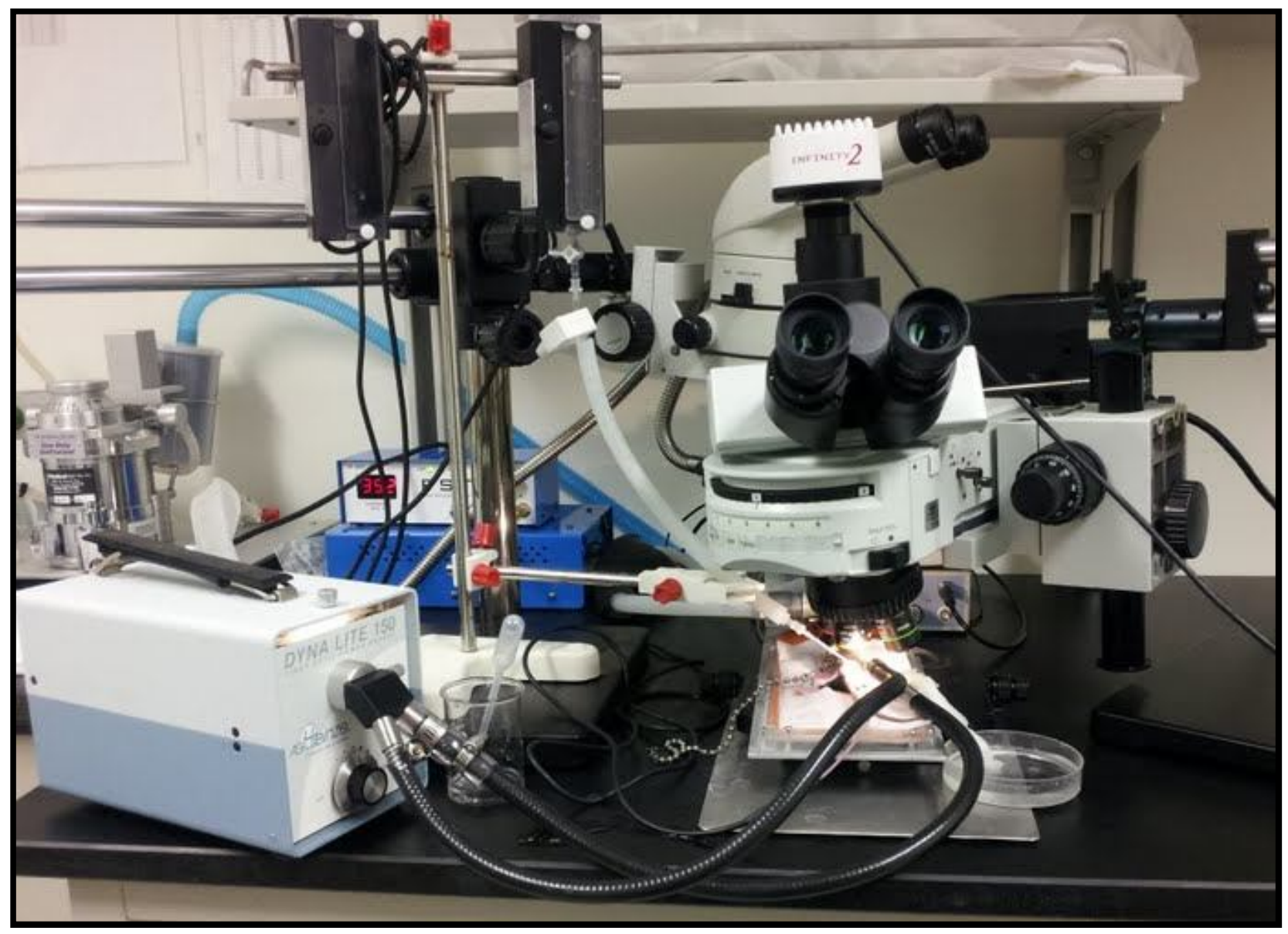

Figure 16: Superfusion experimental setup. Solution flows from a heated syringe, through insulated tubing, and over the mouse hindlimb.

Following equilibration, a baseline image of the profunda femoris was captured. A dosage response curve $\left(10^{-9}-10^{-5} \mathrm{M}\right)$ was obtained for acetylcholine and sodium nitroprusside. Micropipettes were used to transfer the agents to the heated syringe. The arteries were given 5 minutes to equilibrate to the dose administration before images were captured. The process was repeated on the contralateral limb. Measurement order 
between hindlimbs and pharmacologic agents was randomized. Following the experiment, mice were euthanized by cervical dislocation.

\section{IMAGING AND STATISTICAL ANALYSIS}

Infinity imaging software was used to capture images, ImageJ was used to measure vessel diameters, and Microsoft Excel and Minitab were used to perform statistical analysis. Data are presented as mean $\pm \mathrm{SEM}$ with $\mathrm{p} \leq 0.05$ representing statistical significance. Baseline diameter differences were assessed by independent t-test and diameter differences after vasodilator application were assessed by general linear model ANOVA using Tukey interval comparison. 


\section{RESULTS}

Day 7

Acetylcholine

Resting profunda femoris diameter in the operated limb was significantly larger than in the sham 7 days post-ligation (Figure 17B). No significant difference was observed between the diameters of the ligated and sham limbs at all doses of the endothelial-dependent vasodilator, acetylcholine (Figure 17B), although there was a clear trend toward a larger diameter in the ligated limb. Percent change in vessel diameter, a more precise indicator of vascular reactivity when comparing vessels of different resting diameters, was significantly lower in the ligated limb at high acetylcholine doses (Figure 17C), indicating that vasodilation is impaired during the early stages of outward remodeling. These results, however, do not confirm dysfunction of the endothelium, as although acetylcholine acts directly on endothelial cells, vasodilation is still dependent on smooth muscle cell activity. Smooth muscle-dependent vasodilation needs to be assessed in order to determine the dysfunction. 


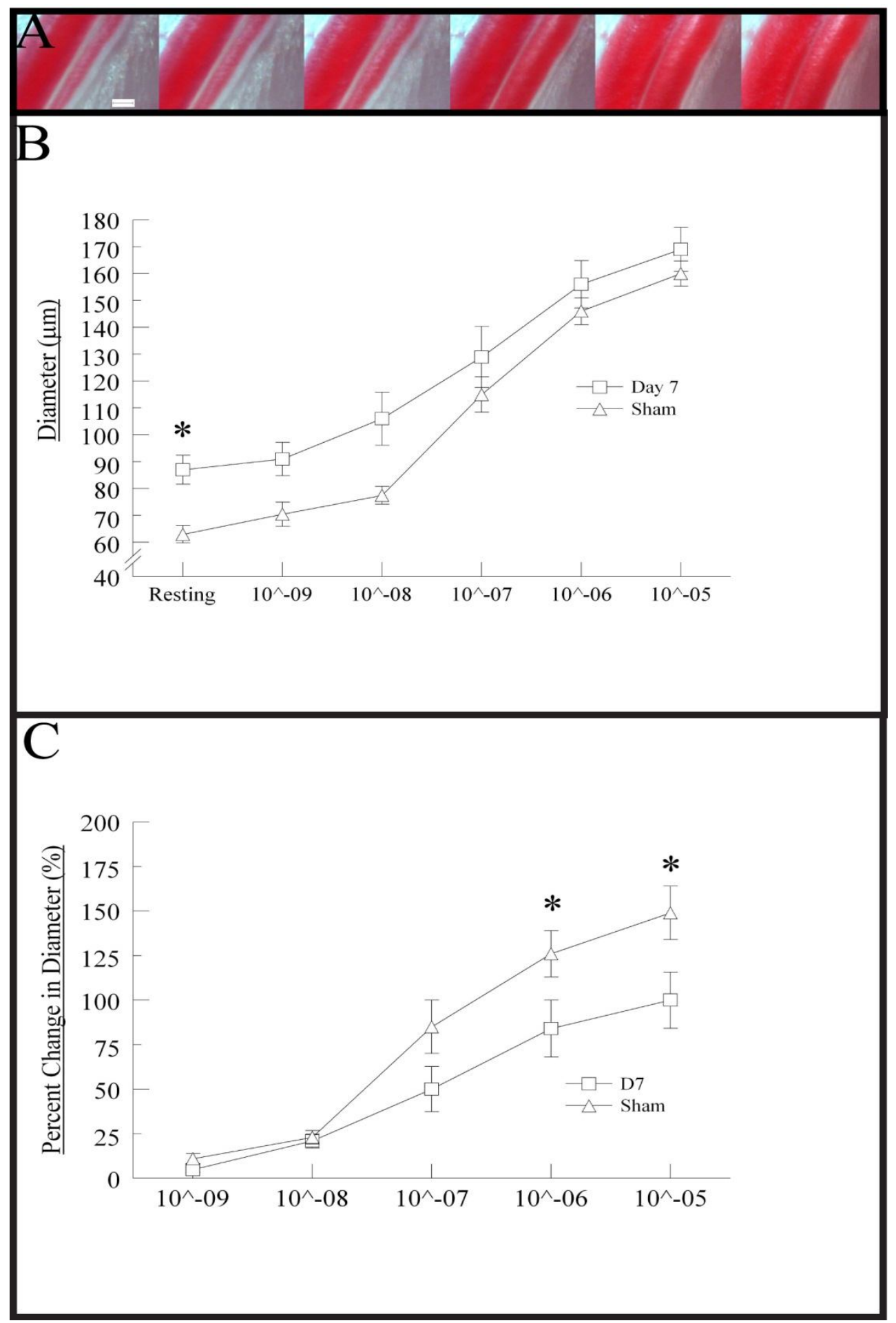

Figure 17: Day 7 Acetylcholine Reactivity. (A) Representative images of the profunda femoris artery in the operated limb at rest and 5 minutes after application of each dose $\left(10^{-9}-10^{-5} \mathrm{M}\right)$ of acetylcholine. (B) The diameter of the profunda femoris in the operated limb was significantly larger at rest $(\mathrm{p}<0.05)$, but was not significantly different after application of each Ach dose ( $\mathrm{p}>0.05)$. (C) Percent change in vessel diameter was significantly different at the $10^{-6}$ and $10^{-5} \mathrm{M}$ dose $(\mathrm{p}<0.05)(\mathrm{n}=7)$. Scale bar is $100 \mu \mathrm{m}$. 


\section{Sodium Nitroprusside}

The results observed with SNP application were very similar to those with acetylcholine. Resting vessel diameter was significantly larger in the ligated limb and no significant difference was observed between the diameters of the ligated and sham limbs at all doses of SNP (Figure 18B), although a clear trend toward a larger diameter in the ligated limb was observed. Percent change in vessel diameter was significantly lower in the ligated limb at high SNP doses (Figure 18C). Both endothelial- and smooth muscledependent vasodilation was impaired at high doses, indicating dysfunction of the smooth muscle cells as both vasodilators ultimately rely on smooth muscle cell function. 


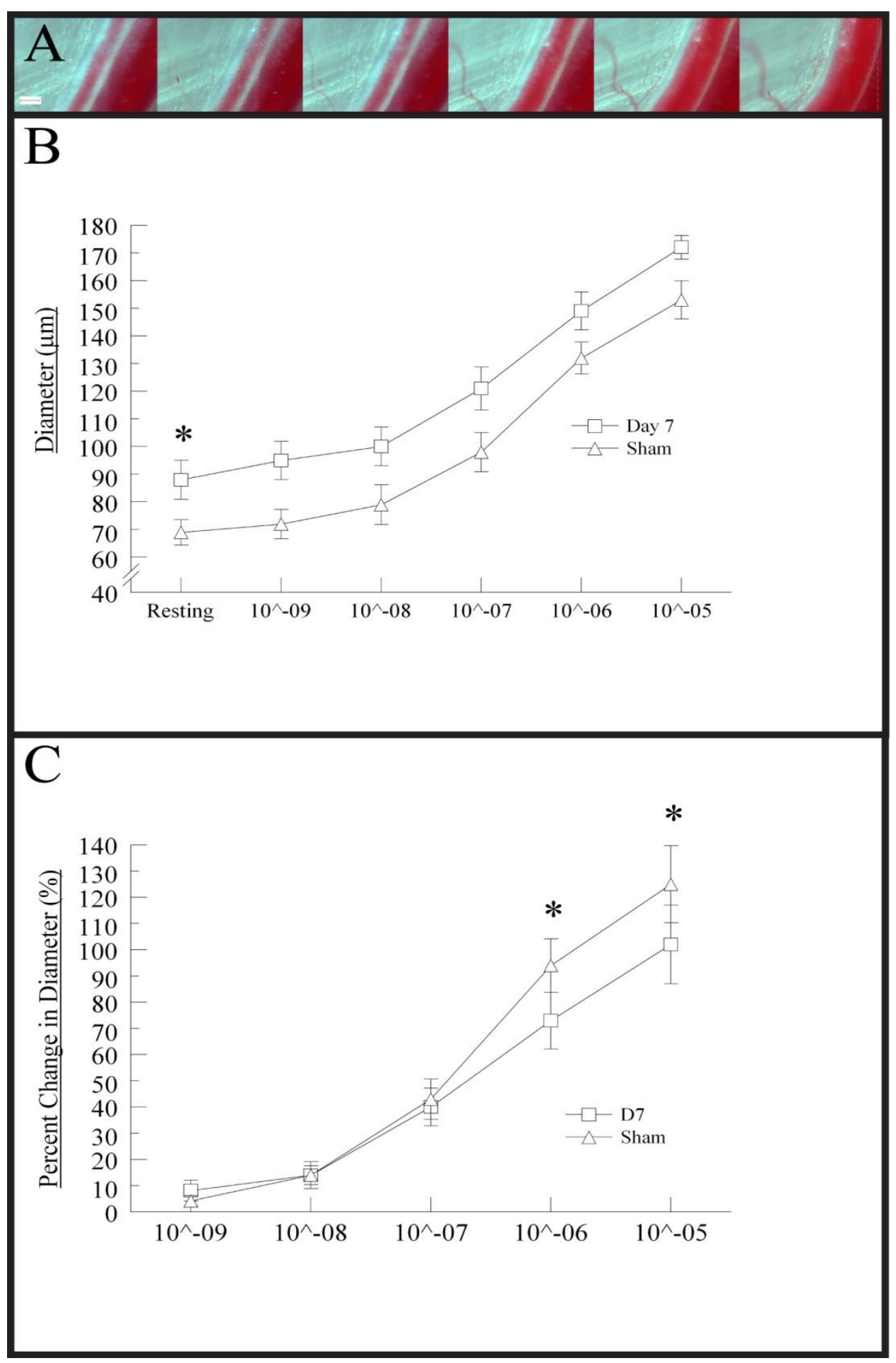

Figure 18: Day 7 Sodium Nitroprusside Reactivity. (A) Representative images of the profunda femoris artery in the operated limb at rest and 5 minutes after application of each dose $\left(10^{-9}-10^{-5} \mathrm{M}\right)$ of SNP. (B) The diameter of the profunda femoris in the operated limb was significantly larger at rest $(\mathrm{p}<0.05)$, but not after application of each SNP dose $(p>0.05)$. (C) Percent change in vessel diameter was significantly different at the $10^{-6}$ and $10^{-5} \mathrm{M}$ dose $(\mathrm{p}<0.05)(\mathrm{n}=7)$. Scale bar is $100 \mu \mathrm{m}$. 
Day 28

Acetylcholine

No significant difference was observed between the diameters of the ligated and sham limbs at all doses of acetylcholine (Figure 19B). Percent change in vessel diameter was also not significantly different at all doses (Figure 19C), indicating restoration of vasodilation. 


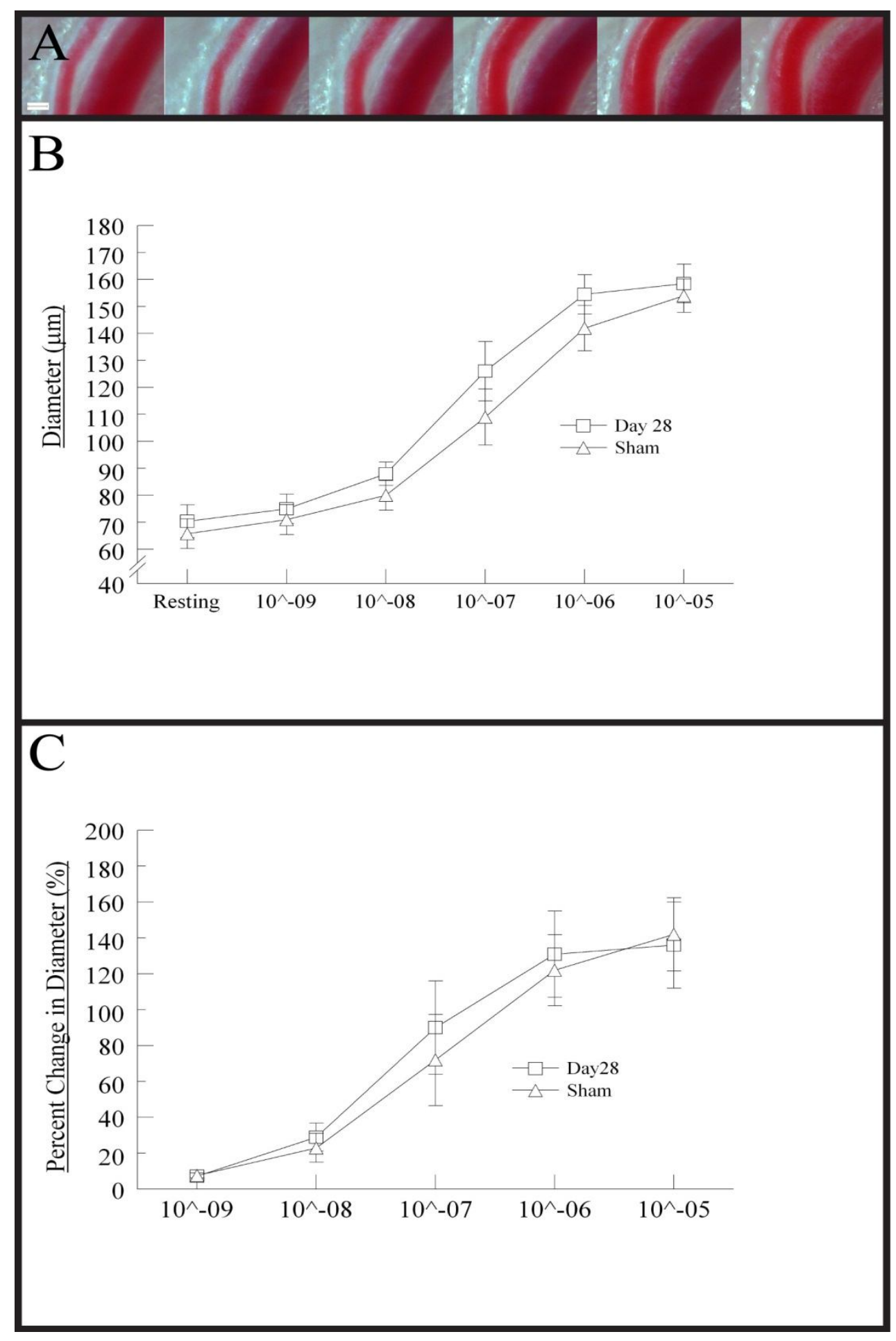

Figure 19: Day 28 Acetylcholine Reactivity. (A) Representative images of the profunda femoris artery in the operated limb at rest and 5 minutes after application of each dose $\left(10^{-9}-10^{-5} \mathrm{M}\right)$ of acetylcholine. (B) The diameter of the profunda femoris in the operated limb was not significantly different at rest or after application of each dose $(p>0.05)$. (C) Percent change in vessel diameter was not significantly different at any dose $(p>0.05)$ $(\mathrm{n}=7)$. Scale bar is $100 \mu \mathrm{m}$. 


\section{Sodium Nitroprusside}

There was a significant difference between the vessel diameters in the ligated and sham limbs at high doses of SNP $\left(10^{-6}\right.$ and $\left.10^{-5} \mathrm{M}\right)$, indicating greater sensitivity to nitric oxide following transient vessel enlargement (Figure 20B). Percent change in vessel

diameter, however, was not significantly different between the limbs at any dose (Figure 20C). 


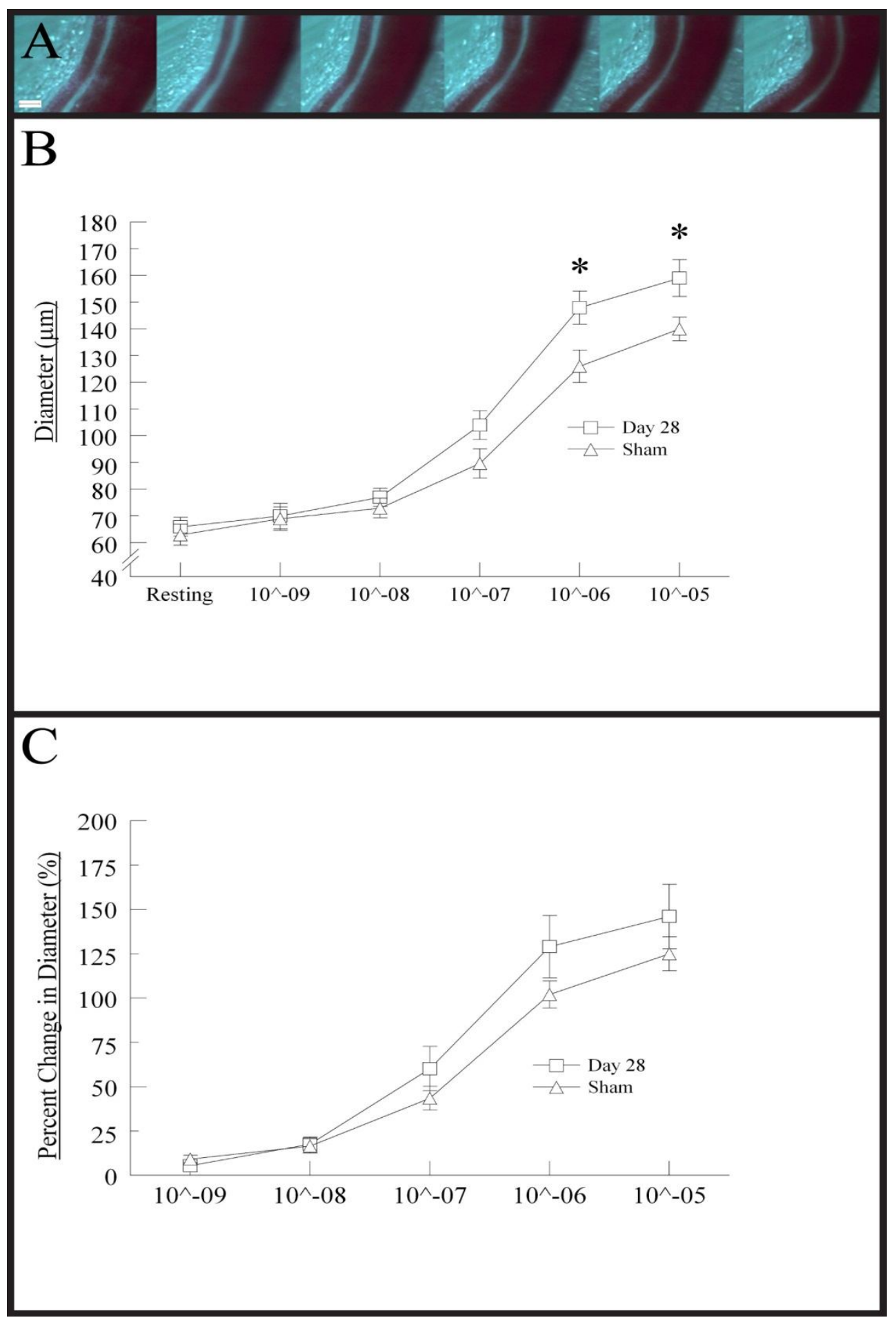

Figure 20: Day 28 Sodium Nitroprusside Reactivity. (A) Representative images of the profunda femoris artery in the operated limb at rest and 5 minutes after application of each dose $\left(10^{-9}-10^{-5} \mathrm{M}\right)$ of SNP. (B) The diameter of the profunda femoris in the operated limb was significantly different at the $10^{-6}$ and $10^{-5} \mathrm{M}$ dose $(\mathrm{p}<0.05)$. (C) Percent change in vessel diameter was not significantly different at any dose $(p>0.05)$ $(\mathrm{n}=7)$. Scale bar is $100 \mu \mathrm{m}$. 


\section{DISCUSSION}

Resting vessel diameter was significantly larger in the ligated limb at day 7 , as was observed in the functional vasodilation experiments (Chapter 3) (Figures 17B \& 18B). At day 7, vasodilation to both acetylcholine and sodium nitroprusside was impaired at high vasodilator concentration, suggesting smooth muscle dysfunction, likely due to cellular repositioning within the vessel wall (Figures $17 \mathrm{C} \& \mathbf{1 8 C}$ ). The ability of smooth muscle cells to relax may be impaired as they reorient themselves to increase vessel diameter due to changes in physical cellular connections or responsiveness to vasodilators.

It is likely that smooth muscle dysfunction is caused by alterations to the extracellular matrix-integrin-cytoskeletal axis during flow-induced remodeling. The sequence of events from vasodilation, to intracellular reorganization of the cytoskeleton, to cellular repositioning, and eventually to a change in passive vessel diameter should be thought of as a continuum as they likely rely on overlapping pathways and depend on the same structural elements [23]. Most of the work done on non-proliferative vascular remodeling has involved chronic vasoconstriction and subsequent inward remodeling. Inward arterial remodeling depends on the concerted activation of enzymes such as tissue-type transglutaminase (thought to induce collagen cross-linking) and focal adhesion kinase (involved in actin polymerization), as well as integrins [23]. Similar interactions are likely involved in outward remodeling brought on by chronic vasodilator stimulus. Figure 21 shows a proposed explanation for impaired vasodilation following chronic vasoconstriction involving the cross-linking of extracellular matrix elements. An 
analogous mechanism may occur during outward remodeling, which could explain the impaired functional and pharmacologic vasodilation observed at day 7.

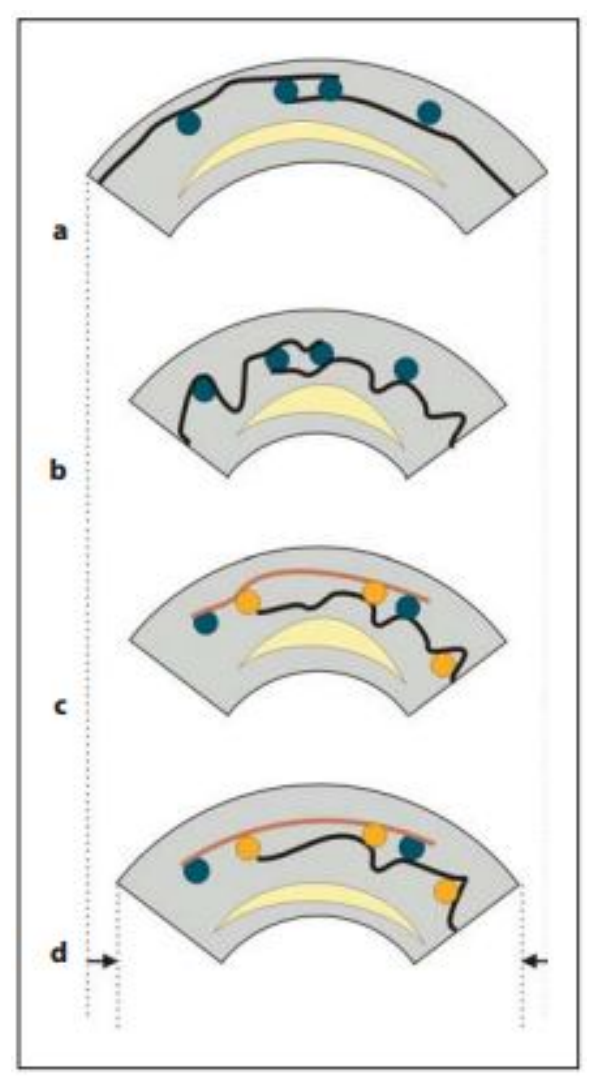

Figure 21: ECM Remodeling. (A) A cross section of a relaxed vessel wall showing a SMC (yellow), matrix fibers (black), and cross-links (blue). (B) SMC contraction leads to matrix unloading. (C) New matrix elements (brown) are formed and new cross-links (orange) are formed between new and existing elements. (D) The new cross-links impair dilation. Adapted from [48].

By day 28 , the only difference between the experimental and sham limb was absolute diameter in response to high SNP doses (Figure 20B). Once the smooth muscle cells (presumably) return to their original positions of increased cellular overlap, they exhibit increased sensitivity to nitric oxide. This altered sensitivity may be explained by the deposition of new extracellular matrix fibers during mechanoadaptation. Synthesis of some ECM fibers, such as collagen, may result in altered ECM/integrin interaction and 
subsequent downregulation of soluble guanylyl cyclase (a factor involved in nitric oxidemediated vasodilation) [49]. Increased sensitivity to nitric oxide donors can occur following periods of decreased nitric oxide availability [50]. Hence, at day 28, vessels may exhibit increased sensitivity to nitric oxide following downregulation of soluble guanylyl cyclase during transient outward remodeling. Percent change in diameter of the vessel was not different between groups (Figures 19C \& 20C). Larger baseline diameter was no longer present in the ligated limb, indicating a reversal in outward remodeling from day 7 (as seen in Chapter 3). The smooth muscle cells likely return to their original positions in response to decreased shear stress and restore their vasodilatory function.

The results of this study are consistent with previous research. Both endothelialand smooth muscle-dependent dilation is impaired in adults at risk of developing atherosclerotic plaques [51,52]. Other vascular reactivity studies aimed at determining the cell type responsible for the impairment in vasodilation in outwardly remodeling vessels observed impaired endothelial- [36, 37] and smooth muscle-dependent dilation, indicating smooth muscle dysfunction $[36,38]$.

One of the limitations to this study is that only two vasodilators were used, acetylcholine and sodium nitroprusside. The next study to perform would be to apply additional vasodilators to the hindlimb following arterial ligation. To better assess what is causing impaired vasodilation, the effect of other vasodilators such as prostanoids and hyperpolarizing factors on vasodilation should be investigated. Different vasodilators are dependent on different cell types and involve different cellular receptors, so by assessing the impact of many dilators, we can better understand the dysfunction. 
Once vasodilation to different vasodilators is assessed in the profunda femoris, the reactivity of the gracilis collateral arterioles in response to these dilators should be assessed. The mechanism of remodeling, and likely the cause of impaired vasodilation, is different in these two types of vessels. Arteriogenesis in the collateral arterioles likely causes dysfunction of the smooth muscle cells as they switch their phenotype. Assessing the reactivity of these vessels to different vasodilators would help determine the cause of impaired vasodilation, if present, during outward remodeling. Multiphoton microscopy in conjunction with fluorescent compounds would need to be used to image the deep vessels.

Some modifications could be made to the superfusion protocol to improve experiment consistency and reproducibility. The flow of gas and superfusion solution varied slightly during each experiment. Flow meters and pumps could be used to provide a controlled gas bubbling rate and solution flow rate. The time needed to perform the experiment could also be reduced by splitting the tip of the insulated tubing to superfuse solution over both hindlimbs at the same time. 


\section{Chapter 5: DISCUSSION}

PAOD is characterized by the buildup of plaque with the vascular wall which physically impedes blood flow and reduces the reactivity of downstream resistance vessels, resulting in ischemic injury in downstream tissues. Blood can be redistributed into collateral vessels, causing increased shear stress and subsequent outward remodeling of the vessels. During outward remodeling, however, vasodilation may be impaired, potentially resulting in ischemic limb pain during ambulation. Impaired vasodilation may be caused by the transition of smooth muscle cell phenotype from contractile to synthetic during arteriogenesis, the repositioning of the cells during mechanoadaptation, or the decrease in endothelial-derived nitric oxide due to shear-induced inflammation.

Stimulating outward vascular remodeling via biologic or pharmacologic agents is a promising therapy for patients with PAOD. However, for these therapies to be effective, their impact on vasoreactivity of the vessels must be understood.

The functional vasodilation and vascular reactivity studies demonstrated that the diameter of the profunda femoris 7 days after femoral occlusion was significantly larger than the sham limb, indicating outward remodeling of the vessel (Figures 13, $17 \& \mathbf{1 8}$ ). Similar observations were documented previously in our lab $[33,53]$. The vasodilatory ability of the profunda femoris in response to gracilis stimulation was impaired at the day 7 time point, likely due to the remodeling process (Figure 13). It is not likely that this impairment is a result of changes in smooth muscle cell phenotype as previous studies observed no cellular proliferation in the profunda femoris following arterial occlusion [33]. 
The enlarged diameter and impaired vasodilatory response of the profunda femoris 7 days after ligation could be attributed to a variety of factors. It is possible that flow-mediated dilation led to increased resting diameter measurements. Occlusion of the femoral artery increases the pressure gradient across the gracilis collateral circuit, resulting in increased blood flow and shear stress in the profunda femoris. Increased shear stress causes the release of vasodilators from the endothelium, which relax the adjacent smooth muscle cells. If the vessel was partially dilated during resting diameter measurement, it would appear as though the vessel had outwardly remodeled, when in actuality, the vessel was just transiently enlarged. This could explain why there is no difference in absolute profunda femoris diameter following vasodilator application (Figures $17 \&$ 18). Flow-mediated dilation would also make it appear as though vasodilation was impaired as resting diameter would be inflated.

However, it is unlikely that flow-mediated dilation would persist for 7 days without triggering a remodeling response. Outward remodeling in response to application of a vasodilator can occur in as little as 3 days [54]. To test whether flow-mediated dilation or remodeling mechanisms caused the increase in profunda femoris diameter at day 7, pressure myography or pharmacologic superfusion experiments could be performed. Profunda femoris arteries could be excised at day 7 from both hindlimbs and isolated in a pressure myograph system. The passive diameter of the vessels would be measured at physiologic pressure in the absence of calcium to eliminate the myogenic response. If structural remodeling of the vessel occurred, the diameter in the ligated limb would be significantly larger. A similar experiment could be performed in vivo using the pharmacologic superfusion protocol employed in Chapter 3. However, instead of 
applying vasodilators to the hindlimb at day 7 , a vasoconstrictor would be used. If outward remodeling occurred, the vessel in the ligated limb would likely constrict to a smaller degree.

If outward remodeling does occur, the increased vessel diameter and impairment in vasodilation is likely due to non-proliferative remodeling, a process termed acute mechanoadaptation. While mechanoadaptation is better documented in inwardly remodeling vessels, an analogous situation likely occurs during outward remodeling. Smooth muscle cells may reorient themselves to decrease their overlap with adjacent cells in response to continual vasodilation stimuli, resulting in an enlarged diameter. The reorientation process itself may impair vasodilation due to changing physical cellular connections or responsiveness to vasodilators. Mechanoadaptation likely involves remodeling of the extracellular matrix-integrin-cytoskeletal axis, which may disrupt cellular relaxation and communication, resulting in impaired dilation. This hypothesis is supported by observations from the vascular reactivity studies, in which vasodilation at day 7 was impaired in response to both endothelial- and smooth muscle- dependent dilators, indicating smooth muscle dysfunction (Figures 17 \& 18).

Not only did the profunda femoris artery enlarge at day 7 , the collateral arterioles in the midzone of the gracilis collateral circuit enlarged in response to the arterial ligation (Figure 15C). The outward remodeling of these vessels is likely attributed to arteriogenesis as proliferating smooth muscle cells are present in these vessels at day 7 [33].

By day 28 post-ligation, profunda femoris artery diameter returned to control resting values and no impairment to functional or pharmacologic vasodilation was 
observed, indicating that vasodilation in feed arteries is transiently impaired following artery occlusion in young, healthy mice (Figures $19 \& 20)$. The collateral arterioles within the gracilis, however, remained enlarged (Figure 15D). A possible explanation for these observations is that the pressure gradient across the gracilis collateral circuit is reduced as the arterioles, which provide the greatest resistance to flow, undergo arteriogenesis. When the collateral arterioles have enlarged enough to normalize shear stress, the pressure gradient across the profunda femoris is reduced, resulting in reduced shear stress and return to control diameter of $\sim 65 \mu \mathrm{m}$. Shear stress in the arterioles likely remains elevated at day 28 as indicated by their maintained increased diameter, while shear stress in the profunda femoris is likely decreased compared to day 7 values. In order for a vessel to outwardly remodel, a "threshold" of shear stress may be needed [42]. It is possible that the shear stress in the profunda femoris decreases below this threshold following collateral arteriole growth, causing the vessel to return to control diameter. Even though the baseline diameter of the profunda femoris was smaller than at day 7 , the dynamic range of the vessel, and its ability to respond to increased metabolic demand via vasodilation, was restored. Furthermore, vasodilation to high doses of SNP was significantly greater in the ligated limb, indicating increased sensitivity to nitric oxide following enlargement.

The outward remodeling process a vessel undergoes is determined by shear stress and vessel size. Normalization of shear stress within a small artery likely requires a smaller degree of remodeling than within collateral arterioles as wall shear stress is inversely proportional to the third power of vessel radius. The transient enlargement and impaired vasodilation of the profunda femoris is likely attributed to mechanoadaptation, 
indicating that small arteries are able to normalize increased wall shear stress via reorientation of smooth muscle cells within the vessel wall. The smaller midzone collaterals, however, undergo arteriogenesis to normalize the increased shear stress via cellular proliferation. The next logical experiment to perform would be to characterize what factors determine if a vessel undergoes arteriogenesis or mechanoadaptation by measuring blood velocity and shear stress in the stem and midzone regions of the collateral circuit at day 7 and 28 by assessing the streak length of jugular-injected microspheres using fluorescent microscopy.

Once the shear stress profiles within the collateral circuit are determined, the next logical study to perform would be to assess mechanoadaptation in the profunda femoris with confocal microscopy 7 and 28 days after femoral ligation. If mechanoadaptation is occurring, a decrease in smooth muscle cell overlap would likely be observed in the ligated limb 7 days after ligation, but overlap would return to control values by day 28 .

Regardless of the position of the smooth muscle cells, the cellular mechanism responsible for impaired vasodilation needs to be determined to identify therapeutic targets. This could be achieved by applying other vasodilators, such as hyperpolarizing factors and prostanoids, to the hindlimb. Changes in vasodilation in response to these factors would help to better identify understand the dysfunction.

Once the remodeling mechanism in the stem region of the collateral circuit is understood, the functionality of the midzone and reentry regions of the circuit should be assessed 7 and 28 days after arterial ligation. Vasodilation in the gracilis feed artery is impaired, but little is known about the intramuscular collaterals. The function of 
collateral arterioles would likely be hindered at day 7 as the vessels undergo arteriogenesis, but would be restored by day 28 following completion of arteriogenesis.

Overall, impaired vasodilation in the mouse hindlimb following arterial ligation was modest and transient, suggesting that young, otherwise healthy animals are capable of rapid and effective remodeling responses. To better understand the mechanism of impaired vasodilation in patients with PAOD, future studies should employ mouse disease models. The majority of patients with PAOD have other comorbidities. Hence, aged, diabetic, hypertensive or hypercholesterolemic mice should be used to better assess how collateral enlargement affects vasodilation in an environment more consistent with the human patient. It is possible that patients with these ailments are able to initiate the outward remodeling process (during which vasodilation is impaired), but are unable to fully remodel $[55,56]$. The findings of this study could potentially aid in developing therapies guided toward restoring activity in collateral vessels. It is crucial to understand how comorbidities impact the impairment observed with arterial occlusion alone. This insight will allow the identification of potential therapeutic targets and support the development of novel therapies to aid these patients. 


\section{REFERENCES}

1. Shanmugasundaram, M., V.K. Ram, U.C. Luft, M. Szerlip, and J.S. Alpert, Peripheral arterial disease--what do we need to know? Clin Cardiol. 34(8): p. 478-82.

2. Selvin, E. and T.P. Erlinger, Prevalence of and Risk Factors for Peripheral Arterial Disease in the United States. Circulation, 2004. 110(6): p. 738-743.

3. Golomb, B.A., T.T. Dang, and M.H. Criqui, Peripheral arterial disease: morbidity and mortality implications. Circulation, 2006. 114(7): p. 688-99.

4. Kasapis, C. and H.S. Gurm, Current approach to the diagnosis and treatment of femoral-popliteal arterial disease. A systematic review. Curr Cardiol Rev, 2009. 5(4): p. 296-311.

5. Health, N.I.o., What Is Peripheral Arterial Disease? http://www.nhlbi.nih.gov/health/health-topics/topics/pad/.

6. Kannel, W.B., D. McGee, and T. Gordon, A general cardiovascular risk profile: the Framingham Study. Am J Cardiol, 1976. 38(1): p. 46-51.

7. Haring, R., T.G. Travison, S. Bhasin, R.S. Vasan, H. Wallaschofski, M.N. Davda, A. Coviello, and J.M. Murabito, Relation between Sex Hormone Concentrations, Peripheral Arterial Disease, and Change in Ankle-Brachial Index: Findings from the Framingham Heart Study. J Clin Endocrinol Metab. 96(12): p. 3724-32.

8. Ostergren, J., P. Sleight, G. Dagenais, K. Danisa, J. Bosch, Y. Qilong, and S. Yusuf, Impact of ramipril in patients with evidence of clinical or subclinical peripheral arterial disease. Eur Heart J, 2004. 25(1): p. 17-24.

9. Hiatt, W., Pathophysiology of Intermittent Claudication in Peripheral Arterial Disease. CardiologyRounds, 2006. 10(1).

10. Crowther, M.A., Pathogenesis of atherosclerosis. Hematology Am Soc Hematol Educ Program, 2005: p. 436-41.

11. Kumar V., A.A., Fausto N., Mitchell R., Robbins Basic Pathology. 8 ed. 2007, Philadelphia: Saunders.

12. Leng, G.C., B. Fowler, and E. Ernst, Exercise for intermittent claudication. Cochrane Database Syst Rev, 2000(2): p. CD000990.

13. Thijssen, D.H., N.T. Cable, and D.J. Green, Impact of exercise training on arterial wall thickness in humans. Clin Sci (Lond). 122(7): p. 311-22.

14. Wang, Y., S. Wang, W.G. Wier, Q. Zhang, H. Jiang, Q. Li, S. Chen, Z. Tian, Y. Li, X. Yu, M. Zhao, J. Liu, J. Yang, J. Zhang, and W. Zang, Exercise improves the dilatation function of mesenteric arteries in postmyocardial infarction rats via a PI3K/Akt/eNOS pathway-mediated mechanism. Am J Physiol Heart Circ Physiol. 299(6): p. H2097-106.

15. Lu, J.T. and M.A. Creager, The relationship of cigarette smoking to peripheral arterial disease. Rev Cardiovasc Med, 2004. 5(4): p. 189-93.

16. Burns, P., S. Gough, and A.W. Bradbury, Management of peripheral arterial disease in primary care. BMJ, 2003. 326(7389): p. 584-588. 
17. Hirsch, A.T., D. Treat-Jacobson, H.A. Lando, and D.K. Hatsukami, The role of tobacco cessation, antiplatelet and lipid-lowering therapies in the treatment of peripheral arterial disease. Vasc Med, 1997. 2(3): p. 243-51.

18. Almahameed, A. and D.L. Bhatt, Contemporary management of peripheral arterial disease: III. Endovascular and surgical management. Cleve Clin J Med, 2006. 73 Suppl 4: p. S45-51.

19. Wolf, G.L., S.E. Wilson, A.P. Cross, R.H. Deupree, and W.B. Stason, Surgery or balloon angioplasty for peripheral vascular disease: a randomized clinical trial. Principal investigators and their Associates of Veterans Administration Cooperative Study Number 199. J Vasc Interv Radiol, 1993. 4(5): p. 639-48.

20. Grundmann, S., J.J. Piek, G. Pasterkamp, and I.E. Hoefer, Arteriogenesis: basic mechanisms and therapeutic stimulation. Eur J Clin Invest, 2007. 37(10): p. 75566.

21. Schirmer, S.H., F.C. van Nooijen, J.J. Piek, and N. van Royen, Stimulation of collateral artery growth: travelling further down the road to clinical application. Heart, 2009. 95(3): p. 191-7.

22. Keeling, A.N., T.J. Carroll, M.M. McDermott, K. Liu, Y. Liao, C.T. Farrelly, W.H. Pearce, and J. Carr, Clinical correlates of size and number of collateral vessels in peripheral artery disease. Vasc Med.

23. Martinez-Lemus, L.A., M.A. Hill, and G.A. Meininger, The plastic nature of the vascular wall: a continuum of remodeling events contributing to control of arteriolar diameter and structure. Physiology (Bethesda), 2009. 24: p. 45-57.

24. Martinez-Lemus, L.A., M.A. Hill, S.S. Bolz, U. Pohl, and G.A. Meininger, Acute mechanoadaptation of vascular smooth muscle cells in response to continuous arteriolar vasoconstriction: implications for functional remodeling. FASEB J, 2004. 18(6): p. 708-10.

25. Schaper, W. and D. Scholz, Factors regulating arteriogenesis. Arterioscler Thromb Vasc Biol, 2003. 23(7): p. 1143-51.

26. Lusis, A.J., Atherosclerosis. Nature, 2000. 407(6801): p. 233-241.

27. van Royen, N., S.H. Schirmer, B. Atasever, C.Y. Behrens, D. Ubbink, E.E. Buschmann, M. Voskuil, P. Bot, I. Hoefer, R.O. Schlingemann, B.J. Biemond, J.G. Tijssen, C. Bode, W. Schaper, J. Oskam, D.A. Legemate, J.J. Piek, and I. Buschmann, START Trial: a pilot study on STimulation of ARTeriogenesis using subcutaneous application of granulocyte-macrophage colony-stimulating factor as a new treatment for peripheral vascular disease. Circulation, 2005. 112(7): $\mathrm{p}$. 1040-6.

28. van Royen, N., J.J. Piek, I. Buschmann, I. Hoefer, M. Voskuil, and W. Schaper, Stimulation of arteriogenesis; a new concept for the treatment of arterial occlusive disease. Cardiovasc Res, 2001. 49(3): p. 543-53.

29. Lederman, R.J., F.O. Mendelsohn, R.D. Anderson, J.F. Saucedo, A.N. Tenaglia, J.B. Hermiller, W.B. Hillegass, K. Rocha-Singh, T.E. Moon, M.J. Whitehouse, and B.H. Annex, Therapeutic angiogenesis with recombinant fibroblast growth factor-2 for intermittent claudication (the TRAFFIC study): a randomised trial. Lancet, 2002. 359(9323): p. 2053-8.

30. Bagher, P. and S.S. Segal, Regulation of blood flow in the microcirculation: role of conducted vasodilation. Acta Physiol (Oxf). 202(3): p. 271-84. 
31. Struthers, K.R., ISCHEMIA IMPAIRS VASODILATION IN SKELETAL MUSCLE RESISTANCE ARTERY in Biomedical Engineering. 2011, California Polytechnic State University, San Luis Obispo San Luis Obispo p. 94.

32. Clifford, P.S. and Y. Hellsten, Vasodilatory mechanisms in contracting skeletal muscle. J Appl Physiol, 2004. 97(1): p. 393-403.

33. Bynum, A.J., Impact of Collateral Enlargement on Smooth Muscle Phenotype, in Biomedical Engineering. 2011, California Polytechnic State University San Luis Obispo: San Luis Obispo. p. 87.

34. D. Neil Granger, E.S., Inflammation and the Microcirculation. 2010, San Rafael: Morgan \& Claypool Life Sciences.

35. Zhang, C., The role of inflammatory cytokines in endothelial dysfunction. Basic Res Cardiol, 2008. 103(5): p. 398-406.

36. Kelsall, C.J., M.D. Brown, and O. Hudlicka, Alterations in reactivity of small arterioles in rat skeletal muscle as a result of chronic ischaemia. J Vasc Res, 2001. 38(3): p. 212-8.

37. Takeshita, S., T. Isshiki, M. Ochiai, K. Eto, H. Mori, E. Tanaka, K. Umetani, and T. Sato, Endothelium-dependent relaxation of collateral microvessels after intramuscular gene transfer of vascular endothelial growth factor in a rat model of hindlimb ischemia. Circulation, 1998. 98(13): p. 1261-3.

38. Takeshita, S., T. Isshiki, H. Mori, E. Tanaka, A. Tanaka, K. Umetani, K. Eto, Y. Miyazawa, M. Ochiai, and T. Sato, Microangiographic assessment of collateral vessel formation following direct gene transfer of vascular endothelial growth factor in rats. Cardiovasc Res, 1997. 35(3): p. 547-52.

39. Waltenberger, J., J. Lange, and A. Kranz, Vascular endothelial growth factor-Ainduced chemotaxis of monocytes is attenuated in patients with diabetes mellitus: A potential predictor for the individual capacity to develop collaterals. Circulation, 2000. 102(2): p. 185-90.

40. Van Belle, E., A. Rivard, D. Chen, M. Silver, S. Bunting, N. Ferrara, J.F. Symes, C. Bauters, and J.M. Isner, Hypercholesterolemia attenuates angiogenesis but does not preclude augmentation by angiogenic cytokines. Circulation, 1997. 96(8): p. 2667-74.

41. Nakae, I., M. Fujita, K. Miwa, K. Hasegawa, Y. Kihara, R. Nohara, S. Miyamoto, K. Ueda, S. Tamaki, and S. Sasayama, Age-dependent impairment of coronary collateral development in humans. Heart Vessels, 2000. 15(4): p. 176-80.

42. Scholz, D. and W. Schaper, Preconditioning of arteriogenesis. Cardiovasc Res, 2005. 65(2): p. 513-23.

43. Yocum, M., ARTERIOGENIC REVASCULARIZATION DOES NOT INDUCE VASCULAR FUNCTION IMPAIRMENT, in Biomedical Engineering. 2009, California Polytechnic State University San Luis Obispo: San Luis Obispo.

44. Kesler, T., Impaired Vascular Reactivity Following Chronic Ischemia in the Arteries of the Mouse Hindlimb, California Polytechnic State University San Luis Obispo.

45. Hudlicka, O., M.D. Brown, S. Egginton, and J.M. Dawson, Effect of long-term electrical stimulation on vascular supply and fatigue in chronically ischemic muscles. J Appl Physiol, 1994. 77(3): p. 1317-24. 
46. Dawson, J.M., I. Okyayuz-Baklouti, and O. Hudlicka, Skeletal muscle microcirculation: the effects of limited blood supply and treatment with torbafylline. Int J Microcirc Clin Exp, 1990. 9(4): p. 385-400.

47. Quyyumi, A.A., D. Mulcahy, N.P. Andrews, S. Husain, J.A. Panza, and R.O. Cannon, 3rd, Coronary vascular nitric oxide activity in hypertension and hypercholesterolemia. Comparison of acetylcholine and substance P. Circulation, 1997. 95(1): p. 104-10.

48. van den Akker, J., M.J. Schoorl, E.N. Bakker, and E. Vanbavel, Small artery remodeling: current concepts and questions. J Vasc Res. 47(3): p. 183-202.

49. De Frutos, S., M. Saura, M. Griera, F.J. Rivero-Vilches, C. Zaragoza, D. Rodriguez-Puyol, and M. Rodriguez-Puyol, Differential regulation of soluble guanylyl cyclase expression and signaling by collagens: involvement of integrinlinked kinase. J Am Soc Nephrol, 2005. 16(9): p. 2626-35.

50. Friebe, A. and D. Koesling, Regulation of nitric oxide-sensitive guanylyl cyclase. Circ Res, 2003. 93(2): p. 96-105.

51. Adams, M.R., J. Robinson, R. McCredie, J.P. Seale, K.E. Sorensen, J.E. Deanfield, and D.S. Celermajer, Smooth muscle dysfunction occurs independently of impaired endothelium-dependent dilation in adults at risk of atherosclerosis. $\mathrm{J}$ Am Coll Cardiol, 1998. 32(1): p. 123-7.

52. Celermajer, D.S., K.E. Sorensen, C. Bull, J. Robinson, and J.E. Deanfield, Endothelium-dependent dilation in the systemic arteries of asymptomatic subjects relates to coronary risk factors and their interaction. J Am Coll Cardiol, 1994. 24(6): p. 1468-74.

53. Machado, M., Impaired Resistance Artery Reactivity Following Arteriogenesis, in Biomedical Engineering. 2011, California Polytechnic State University: San Luis Obispo.

54. Sorop, O., E.N. Bakker, A. Pistea, J.A. Spaan, and E. VanBavel, Calcium channel blockade prevents pressure-dependent inward remodeling in isolated subendocardial resistance vessels. Am J Physiol Heart Circ Physiol, 2006. 291(3): p. H1236-45.

55. Tirziu, D., K.L. Moodie, Z.W. Zhuang, K. Singer, A. Helisch, J.F. Dunn, W. Li, J. Singh, and M. Simons, Delayed arteriogenesis in hypercholesterolemic mice. Circulation, 2005. 112(16): p. 2501-9.

56. Yang, H.T., Effect of Aging On Angiogenesis and Arteriogenesis Cardiology, 2007. 3: p. 65-74. 


\section{APPENDIX}

\section{Femoral Artery Ligation Protocol}

Date

Mouse Information

DOB:

Sex:

Tag:

Genotype/strain

Cage:

\section{Materials}

Sterilize- autoclave or flash autoclave

$\begin{array}{ll}\text { 1. } & \text { forceps (2) } \\ \text { 2. } & \text { fine forceps (2) } \\ \text { 3. } & \text { ultrafine forceps (1) } \\ \text { 4. } & \text { fine scissors (1) } \\ \text { 5. } & \text { microscissors spring loaded (1) }\end{array}$

Pre-sterilize in autoclave

\begin{aligned} 6. & cotton gauze (2) \\ 7. & cotton swabs (12) \\ 8. & 6.0 silk suture $(2 \times 1$-inch) \\ \hline 9. & needle holder (1) \end{aligned}

Obtained in surgery suite

10. sterile Petri dishw/ sterile saline

11. sterile gloves

12. sterile 7.0 prolene suture

13. heat-cautery

14. FST heat pad w/rectal probe

15. heat pad

16. recovery bin \& weigh boat

17. depilatory cream

18. non-sterile cotton swabs

19. non-sterile cotton gauze

20. isolation mask \& cap

21. analgesic (Buprenorphine)

\section{Surgery preparation}

22. Spray surgery area with Nolvasan

23. Weigh animal in weight boat

24. Place animal in anesthesia box

25. Open the oxygen cylinder and set

anesthesia-machine flow meter to $\sim 31 \cdot \mathrm{min}^{-1}$

26. Anesthetize animal $w / 5 \%$ isoflurane

27. Affix non-rebreathing circuit to bench-top with tape

28. Reduce flow rate to $0.5-1.0 \mathrm{l} \cdot \mathrm{min}^{-1}$ and the isoflurane to $1-3 \%$

29. Apply ear tag high on left ear

30. Lay animal supine with nose in nose-cone

31. Shave hair on the right hindlimb \& lower abdomen with clippers

32. Remove excess hair with depilatory cream

33. Spray right hindlimb with Nolvasan

34. Return animal to anesthesia box
Initials

35. Apply $4 \times 4$ gauze sponge to heat pad to protect animal from excessive heat

36. Affix non-rebreathing circuit to surgery table w/ chemistry clamp

37. Lay animal supine on circulating heat pad w/ nose in nose-cone

38. Insert rectal probe and set thermo-controller to $35^{\circ} \mathrm{C}$

39. Apply veterinary ointment to eyes to avoid drying during procedure

40. Apply veterimary ointment to anus and place rectal probe $\sim 1 \mathrm{~cm}$ into anus to monitor corebody temperature

Surgery

41. Make a small incision on the middle, medial aspect of the left thigh

42. Extend the incision up to the abdominal wall

43. Blunt dissect the subcutaneous connective tissue to maximize surgical exposure

44. Use cautery to remove fat pad overlying femoral a-v pair \& cauterize epigastric aypair

45. Blunt dissect the femoral artery from the neurovascular bundle just downstream from the deep femoral branch

46. Tie off the femoral artery \& vein with 6.0 silk suture, just downstream to the deep femoral branch

47. Use 6.0 polypropylene suture to close the skin

48. Make a small incision on the middle, medial aspect of the right thing

49. Extend the incision up to the abdominal wall

50. Blunt dissect the subcutaneous connective tissue to maximize surgical exposure

51. Use 6.0 polypropylene suture to close the skin

Post-Surgical

52. Give the animal an subcutaneous injection of buprenorphine $(0.075 \mathrm{mg} / \mathrm{kg})$

53. Place the animal in the recovery bin, on a blue bench cover, above a heat pad and allow to recover

54. Tum flow meter down to 0 , tum off isoflurane, and close the oxygen cylinder

55. Indicate surgery on cage card 
Functional Vasodilation Protocol

Date

Mouse Information
DOB:-
Sex:-
Tag
Genotyperstrain
Cage:

\section{Materiab}

Instruments

$\begin{array}{ll}\text { 1. } & \text { forceps (2) } \\ \text { 2. } & \text { fine forceps (2) } \\ \text { 3. } & \text { ultrafine forceps (1) } \\ \text { 4. } & \text { fine scissors (1) }\end{array}$

Obtained in surgery suite

$$
\begin{array}{ll}
\text { 5. } & \text { Petri dish w/ PBS } \\
\text { 6. Plastic wrap } \\
\text { 7. } \\
\text { 8. } \\
\text { 8. } \\
\text { 9. } \\
\text { 10. noves heat pad w/ rectal probe } \\
\text { 11. non-sterile cottion swabs } \\
\text { 11. Tungsten stimulating microelectrodes }
\end{array}
$$

\section{Experiment preparation}

12. Place animal in anes thesia box

13. Open theoxygen cylinder and set anesthesiamachine flow meter to $\sim 31 \cdot \min ^{-1}$

14. Anesthetize animal $w / 5 \%$ is oflurane

15. Weigh animal in weigh boat

16. Reduce flow rate to $0.8-1.01 \cdot \min ^{-1}$ and the isofloaneto $1-3 \%$

17. Move mouse to nose-cone and remove excess hair with clippers and depilatory crean

18. L ay animal supine on gruze pad on circulating heat pad wi nose in nosecone

19. Insert rectal probe and set thermo-controller to $35^{\circ} \mathrm{C}$

20. Gently tape hind paws to gruzepad

Measurement preparation

21. Make a small incision on the middle medial aspect of the left thigh

22. Extend the incision proximally to the abdominal wall

23. Apply PBS to prevent desiccation

24. Blunt dissect thesuboutaneous connective tissue to maximize surgical exposuse

25. Maximize visibility of muscular branch

26. Tum on PowerLab system and stimulator panel and insert stimulating electrodes

27. Place stimulating electrodes ( $4 \mathrm{~mm}$ apart) electrode on the center of the gracilis anterior and secure electrodes using clay

28. Open file in blood pressure measurement file in Lab Chart 6

29. Set frequency to $1 \mathrm{~Hz}$, duration to $200 \mathrm{\mu s}$, current to $\operatorname{lm} A$
Initials

30. Perform brief set of contractions to ensure strong muscle contraction

31. Place mineral oil and a small piece of plastic wrap over exposed areas

\section{Olympus BKFM}

32. Open Infmity software

33. Position Olympus BXFM over distal profunda femoris just before the vessel passes under the gracilis and adjut for best resolution.

34. Allow 30 mimute equilibration period

35. Capture image of profunda before stimulation

36. Change stimilation frequency to $8 \mathrm{~Hz}$

37. Stimulate musde for $90 \mathrm{sec}$

38. Capture image immediately after stimulation and each subsequent mimite until vessel returns to baseline

39. Repeat procedure on contralateral limb

Analysis

40. Open Image and calibrate using micrometer 41. Measure vessel diameter using Ime tool Record results in provided table

Post-Surgical

42. Cervical dislocation to euthanize animal

-43. Place animal in biohazard bag in freecer

\section{Notes}

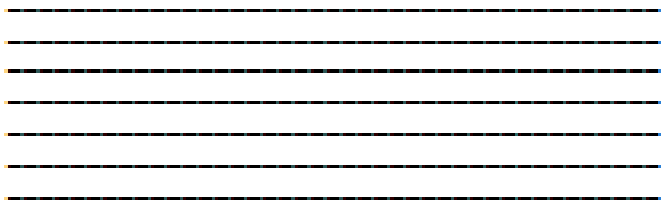




\begin{tabular}{|c|l|}
\hline Experimentalimb & Diameter \\
\hline $\begin{array}{c}\text { Resting: } \\
\text { mus1 }\end{array}$ & \\
\hline $\begin{array}{c}\text { PostStim: } \\
\text { mus2 }\end{array}$ & \\
\hline mus3 & \\
\hline mus4 & \\
\hline mus5 & \\
\hline mus6 & \\
\hline mus7 & \\
\hline mus8 & \\
\hline mus9 & \\
\hline mus10 & \\
\hline mus11 & \\
\hline mus12 & \\
\hline mus13 & \\
\hline mus14 & \\
\hline mus15 & \\
\hline
\end{tabular}

\begin{tabular}{|c|l|}
\hline $\begin{array}{c}\text { ControlLimb } \\
\text { Resting: } \\
\text { sham } 1\end{array}$ & Diameter \\
\hline $\begin{array}{c}\text { PostStim: } \\
\text { sham } 2\end{array}$ & \\
\hline sham3 & \\
\hline sham4 & \\
\hline sham5 & \\
\hline sham6 & \\
\hline sham7 & \\
\hline sham8 & \\
\hline sham9 & \\
\hline sham10 & \\
\hline sham11 & \\
\hline sham12 & \\
\hline sham13 & \\
\hline sham14 & \\
\hline sham15 & \\
\hline
\end{tabular}




\section{Superfusion Protocol}

Date

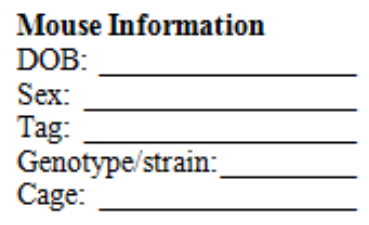

\section{Materials}

\begin{tabular}{|c|c|}
\hline 1. & forceps (2) \\
\hline 2. & fine forceps (2) \\
\hline 3. & ultrafine forceps (1) \\
\hline 4. & fine scissors (1) \\
\hline 5. & $60 \mathrm{ml}$ syringe \\
\hline 6. & Volumetric flask \\
\hline 7. & KimWipes \\
\hline 8. & Reservoir (Petri dish) \\
\hline 9. & Vacuum pump evacuation flask \\
\hline 10. & Vasodilator (Äch \& SNP) \\
\hline 11. & 10XPSS \\
\hline 12. & $10 \mathrm{X} \mathrm{NaHCO}$ \\
\hline 13. & non-sterile cotton swabs \\
\hline 14. & non-sterile cotton gauze \\
\hline 15. & $100 \mathrm{ml}$ Graduated cylinder \\
\hline 16. & Micropipettes \\
\hline 17. & pH meter \\
\hline
\end{tabular}

\section{Instrument Preparation}

18. Turn on water bath to $50^{\circ} \mathrm{C}$

19. Transfer $50 \mathrm{ml}$ of $10 \mathrm{x}$ PSS into $1 \mathrm{~L}$ volumetric flask

20. Transfer $50 \mathrm{ml}$ of $10 \times \mathrm{NaHCO}_{3}$ into $1 \mathrm{~L}$ volumetric flask

21. Fill volumetric flask to $1 \mathrm{~L}$ with $18 \mathrm{M} \Omega$ $\mathrm{H}_{2} \mathrm{O}$

22. Place volumetric flask into water bath and continually bubble with $5 \% \mathrm{CO}_{2}$ $95 \% \mathrm{~N}_{2}$

23. Use $\mathrm{pH}$ meter to ensure $\mathrm{pH}$ of $\sim 7.4$

24. Turn on syringe heater, fill with $50 \mathrm{ml}$ of $1 \times$ PSS, and continually bubble with $5 \%$ $\mathrm{CO}_{2}-95 \% \mathrm{~N}_{2}$

25. Place thermistor in syringe heater

26. Thaw vasodilator

\section{Surgery preparation}

27. Place animal in anesthesia box

28. Open the oxygen cylinder and set anesthesia-machine flow meter to $\sim 3$ $1 \cdot \mathrm{min}^{-1}$
Initials

29. Anesthetize animal $\mathrm{w} / 5 \%$ isoflurane

30. Reduce flow rate to $0.8-1.01 \cdot \mathrm{min}^{-1}$ and the isoflorane to $1-3 \%$

31. Weigh animal in weight boat

32. Lay animal supine on preparation bench in nose-cone

33. Use trimming clippers \& depilatory cream to remove hair on the medial aspect of the hindlimb

34. Transfer mouse to stage on FST heat pad

35. Insert rectal probe and set thermocontroller to $35^{\circ} \mathrm{C}$

36. Place reservoir under tip of tubing

37. Open stopcock on delivery tubing to check flow rate of superfusion solution and use thermistor to check temperature

38. Adjust flow rate or temperature to achieve flow rate of $\sim 2 \mathrm{ml} \cdot \mathrm{min}^{-1}$ and $\sim 35^{\circ} \mathrm{C}$ at tip

\section{Measurement preparation}

39. Make a small incision on the middle, medial aspect of the left thigh

40. Extend the incision up to the abdominal wall

41. Blunt dissect the subcutaneous connective tissue to maximize surgical exposure

42. Apply PBS as needed

43. Maximize visibility of muscular branch

Intravital Microscopy

44. Recheck flow rate and temperature of superfusion solution

45. Position tubing over mouse hindlimb

46. Place KimWipe on distal end of hindlimb to wick solution to reservoir

47. Open Infinity software

48. Position Olympus BXFM over distal profunda femoris just before the vessel passes under the gracilis and adjust for best resolution

49. Allow 30 minute equilibration period

50. Capture baseline image of the profunda

51. Transfer PSS from volumetric flask to syringe until it contains $50 \mathrm{ml} \&$ close stopcock

52. Add first dose of vasodilator agent (10E09) 
Date__Intravital Microscopy with Superfusion

Initials

53. Allow solution to flow over hindlimb for 5 minutes and capture image

54. Open stopcock and refill $60 \mathrm{ml}$ syringe to $50 \mathrm{ml}$ before re-closing

55. Empty reservoir using vacuum pump as needed

56. Repeat for next 4 doses of vasodilator (10E-08-10E-05)

57. Repeat procedure on the contralateral limb

58. Repeat same procedure on both limbs using the other vasodilator

Analysis

59. Open Image J and calibrate using micrometer

60. Measure vessel diameter using line tool

61. Record results in provided table

Post-Experiment

62. Cervical dislocation to euthanize animal

63. Place animal in biohazard bag in freezer

64. Rinse all glassware and tubing with water, then $3 \mathrm{MHCL}$, then distilled water, then $18 \mathrm{M} \Omega \mathrm{H}_{2} \mathrm{O}$ 
Date

Acetylcholine

\begin{tabular}{|l|l|}
\hline Experimental Limb & Diameter $(\mu \mathrm{m})$ \\
\hline Baseline & \\
\hline $10 \mathrm{E}-09$ & \\
\hline $10 \mathrm{E}-08$ & \\
\hline $10 \mathrm{E}-07$ & \\
\hline $10 \mathrm{E}-06$ & \\
\hline $10 \mathrm{E}-05$ & \\
\hline
\end{tabular}

\begin{tabular}{|l|l|}
\hline Sham Limb & Diameter $(\mu \mathrm{m})$ \\
\hline Baseline & \\
\hline $10 \mathrm{E}-09$ & \\
\hline $10 \mathrm{E}-08$ & \\
\hline $10 \mathrm{E}-07$ & \\
\hline $10 \mathrm{E}-06$ & \\
\hline $10 \mathrm{E}-05$ & \\
\hline
\end{tabular}

\begin{tabular}{|l|l|}
\hline Sham Limb & Diameter $(\mu \mathrm{m})$ \\
\hline Baseline & \\
\hline $10 \mathrm{E}-09$ & \\
\hline $10 \mathrm{E}-08$ & \\
\hline $10 \mathrm{E}-07$ & \\
\hline $10 \mathrm{E}-06$ & \\
\hline $10 \mathrm{E}-05$ & \\
\hline
\end{tabular}

Notes

\begin{tabular}{|l|l|}
\hline Experimental Limb & Diameter $(\mu \mathrm{m})$ \\
\hline Baseline & \\
\hline $10 \mathrm{E}-09$ & \\
\hline $10 \mathrm{E}-08$ & \\
\hline $10 \mathrm{E}-07$ & \\
\hline $10 \mathrm{E}-06$ & \\
\hline $10 \mathrm{E}-05$ & \\
\hline
\end{tabular}

$\overline{ } \overline{ } \overline{ }$




\section{Gracilis Anterior Dissection Protocol}

Date

\begin{tabular}{ll} 
Mouse Information \\
DOB: \\
Sex: \\
Tag: \\
Genotype/strain: \\
Cage: \\
Materials \\
1. & Forceps (1) \\
\hline 2. & 5-45 forceps (1) \\
\hline 3. & Iris scissors (1) \\
\hline 4. & Cautery \\
\hline 5 & Pipette \\
\hline 6 & $4 \%$ paraformaldehyde \\
\hline & Plastic conical (2)
\end{tabular}

Gracilis Tissue Dissection

Initials

\section{Dissection}

8. Using 5-45 forceps, blunt dissect the anterior and posterior gracilis muscles away from each other

9. Blunt dissect the anterior muscle from the underlying tissue

10. Pipette $4 \%$ paraformaldehyde over the tissue

11. Observe 20 minute waiting period to allow for tissue fixation

12. Cauterize the femoral artery distally then proximally to the gracilis anterior

13. Repeat (14) with the profunda femoris

14. Cut the anterior gracilis laterally to the femoral artery and medially to the profunda femoris

15. Place muscle in labeled conical (Name, date, limb, muscle)

16. Fill conical with PBS and store in a $4^{\circ} \mathrm{C}$ refrigerator

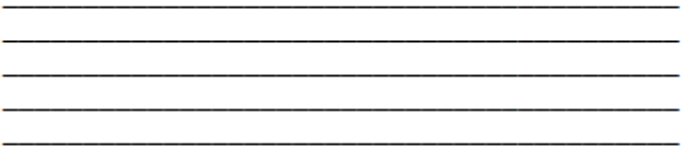




\section{Gracilis Whole Mount Immunostaining Protocol}

Date

Mouse Information
DOB:
Sex:-
Tag:-
Genotype/strain:
Cage:

\section{Materials}

1. Sylgard@ 184 Silicon Elastomer (Cat \#: NC0004554, Fisher Scientific)

2. 24-well culture plates (Cat\#: 3738 , Coming Incorporated)

3. $\mathrm{PBS}$

4. $0.1 \%$ Saponin (Cat\#: 47036 , Sigma-Aldrich)

5. $2 \%$ Bovine Serum Albumin (Cat\# B6917, Sigma Aldrich)

6. Monoclonal Anti-Alpha Smooth Muscle Actin, Cy3 Conjugate (Cat\#: C6198, Sigma-Aldrich)

7. Fluoroshield Mounting Medium with DAPI (Catf: ab104139, Abcam)

8. Slides

9. Coverslips

10. Parafilm

11. Aluminum foil

\section{Sylgard-coated Plate Preparation}

1. Prepare sylgard-coated plates at least 3 days prior to staining.

2. Mix 10:1 sylgard elastomer to curing agent in a $50 \mathrm{~mL}$ conical tube. (Note: sylgard is extremely viscous. Cut tip of pipette to create a larger opening and pipette very slowly, changing tips often).

3. Add 500-750 uL of sylgard mix to each well of a 24 well plate.

4. Allow sylgard to harden at room temperature for 48 hours.

\section{Staining}

1. Using forceps, remove muscle from PBS (stored in microcentrifuge tube at $4^{\circ} \mathrm{C}$ ) and place in a single well of a 24-well sylgard-coated plate.

2. Prepare antibody solution containing 1:200 1A4 clone (alpha-smooth muscleactin, Cy3 conjugate) in $0.1 \%$ saponin (reconstituted in PBS), $2 \%$ BSA (reconstituted in PBS) in PBS.

3. Incubate muscle in antibody solution for 3 nights ( 72 hours) at $4^{\circ} \mathrm{C}$ ). (Note: Critical step-3 nights crucial for bright staining) by gently pipetting solution over muscle.)
4. Wash in $0.1 \%$ saponin in PBS $3 x \overline{\text { for } 20 \text { minutes }}$ at room temperature. Wrap plate in parafilm and cover with foil during each wash.

5. Wash in plain PBS for 30 minutes. Wrap plate in parafilm and cover with foil during each wash.

6. Remove muscle from well using forceps and place on a slide.

7. Add 1-2 drops of mounting media and place coverslip over muscle.

8. Store slides at $4^{\circ} \mathrm{C}$ wrapped in foil between imaging.

Imaging

1. Image using a standard fluorescent microscope. (Cy3 excitation: $550 \mathrm{~nm}$, emission: $570 \mathrm{~nm}$ )

\section{Notes}

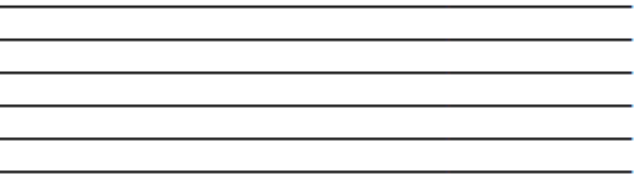

